University of Redlands

\title{
Vector-based Mobility Modeling
}

A Major Individual Project submitted in partial satisfaction of the requirements for the degree of Master of Science in Geographic Information Systems

by

Shreyas S. Shinde

Douglas M. Flewelling, Ph.D., Committee Chair

Mark P. Kumler, Ph.D.

December 2013 
Vector-based Mobility Modeling

Copyright (C) 2013

by

Shreyas S. Shinde 
The report of Shreyas S. Shinde is approved.

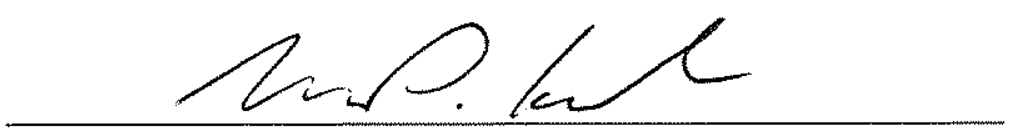

Mark P. Kumler, Ph.D.

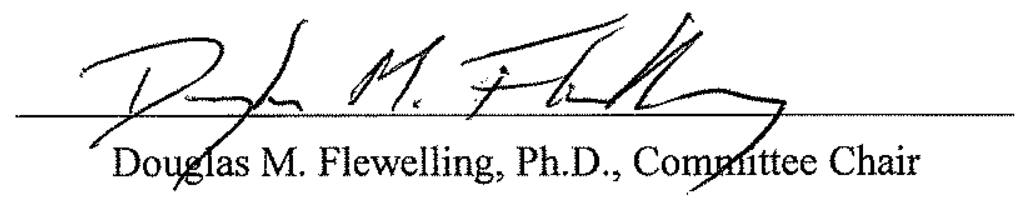

December 2013 



\section{Acknowledgements}

My parents have had the greatest influence on my life and I am what I am because of them. I wouldn't have enrolled into the MS-GIS program if it weren't for their boundless encouragement and faith. I am grateful to my employers at Esri for sponsoring my education, and to my colleagues for supporting me and patiently accommodating my schedule for the past two years. I am thankful to my advisor Dr. Douglas Flewelling, the staff and faculty in the MS-GIS department, for their abundant bytes of wisdom and enlightenment. I am indebted to Debra Riley and Ruben Ortiz who took care of all the little details and made sure that I had access to everything that I needed to learn and complete my program.

Finally, none of this would have been possible without my wife, Prachi's, support, cooperation and her many sacrifices. Prachi, you make everything worthwhile. 



\section{Abstract \\ Vector-based Mobility Modeling}

by

Shreyas S. Shinde

When a large number of routes pass through a narrow region or constricted space on a terrain they create bottlenecks, affecting the flow of goods and people through the region. These regions become important in defense, security and rescue operations because of their ability to intercept and influence many routes simultaneously. This paper describes a novel implementation for finding such regions on a terrain through a suite of Pythonbased geoprocessing tools for ArcMap that apply least-cost path analysis and Monte Carlo simulation to generate a large number of routes on a raster-based cost surface. The implementation is made efficient by resampling the cost surface to a configurable vector grid and by running the simulations using multi-processing. By plotting all the simulated routes on a map, bottlenecks are highlighted. The result is a powerful suite of tools that can be used for planning of rescue missions and troop mobility operations. A hypothetical use case of finding the best paths for an infantry platoon moving across a terrain to its destination is presented in this paper. A cost surface using multi-criteria evaluation is developed and provided as input to the simulation tools which generate hundreds of possible routes between the platoon's starting point and final destination, identifying areas of the terrain that could become possible bottlenecks or ambush points. 



\section{Table of Contents}

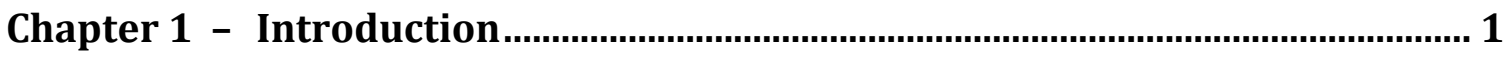

1.1 Client

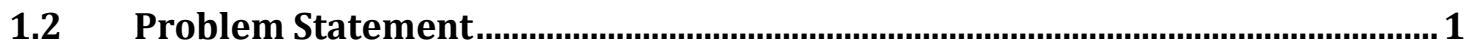

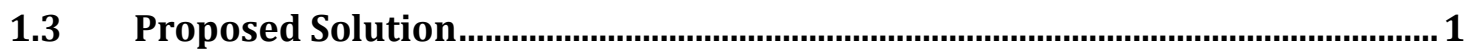

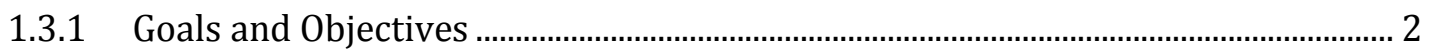

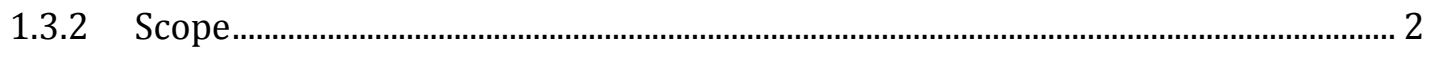

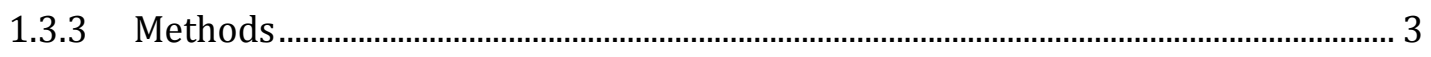

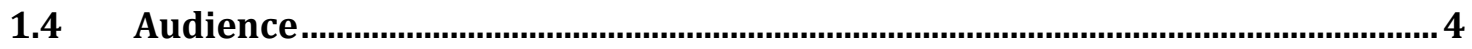

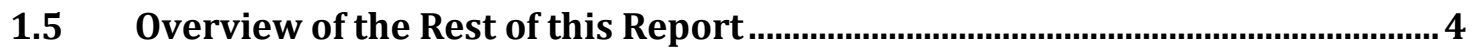

Chapter 2 - Background and Literature Review ….............................................. 7

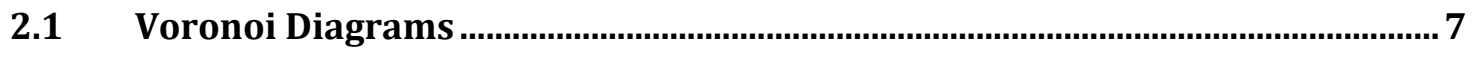

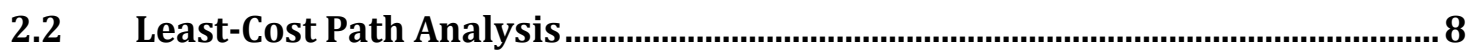

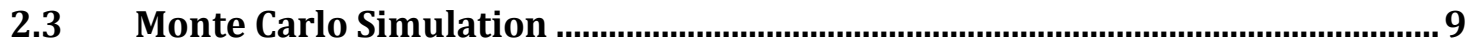

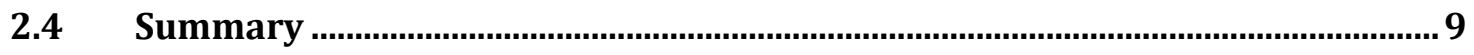

Chapter 3 - Systems Analysis and Design ...........................................................11

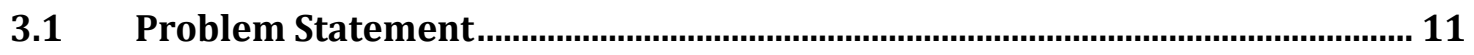

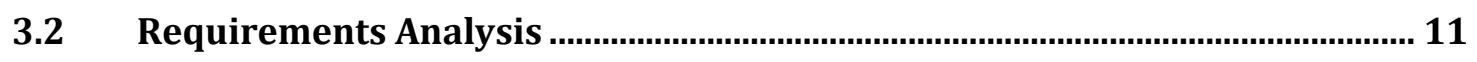

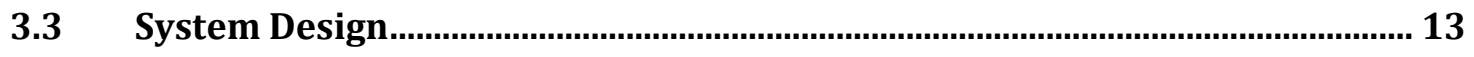

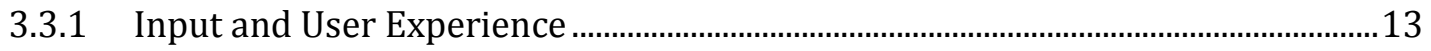

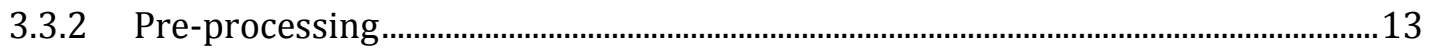

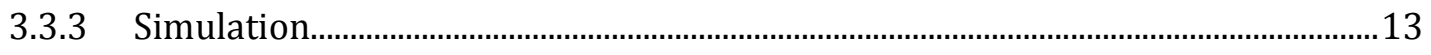

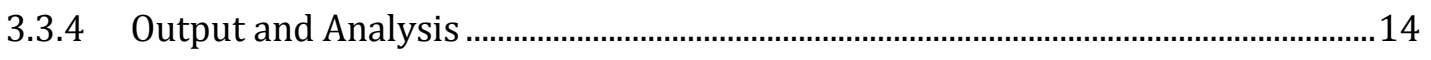

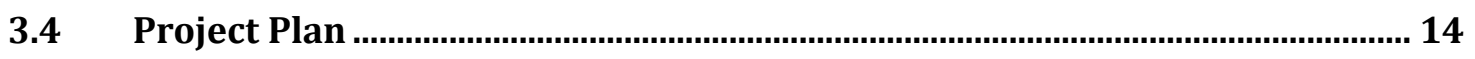


3.5 Summary

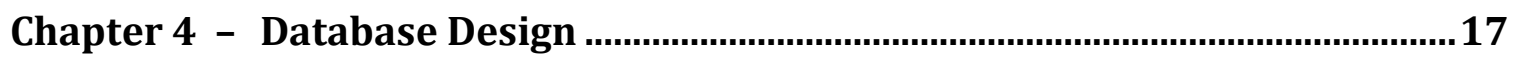

4.1 Conceptual Data Model............................................................................................... 17

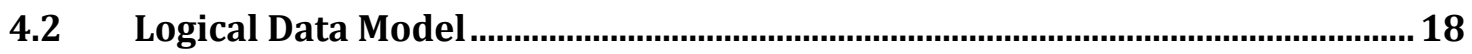

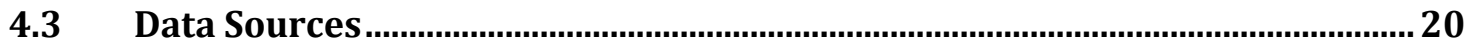

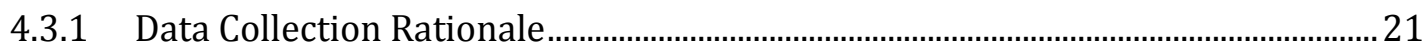

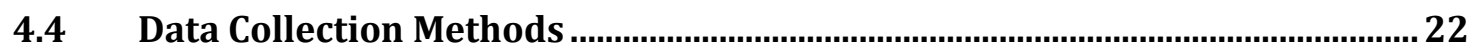

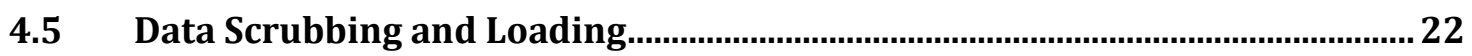

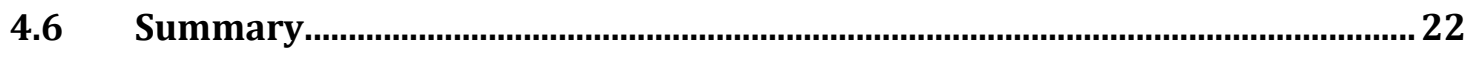

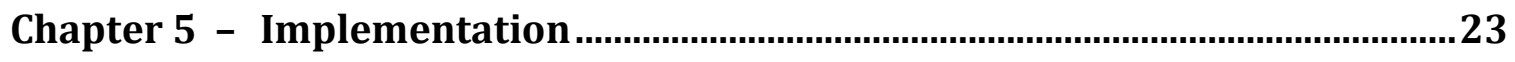

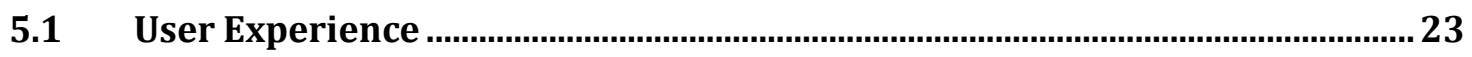

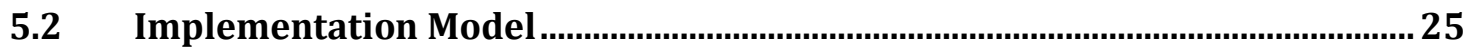

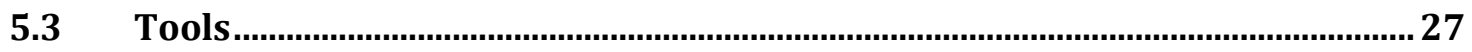

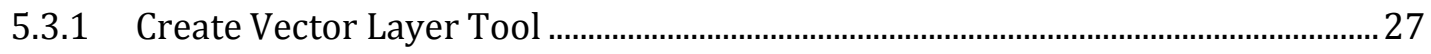

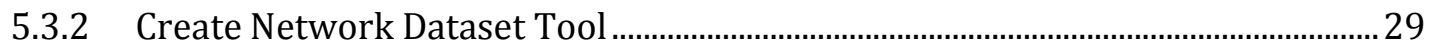

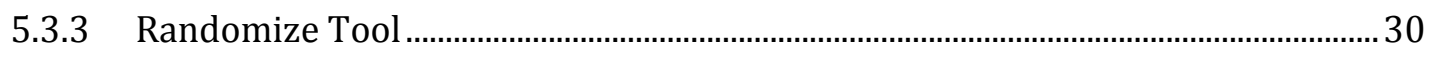

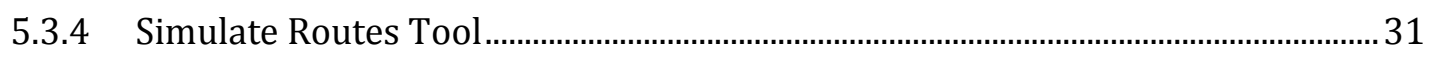

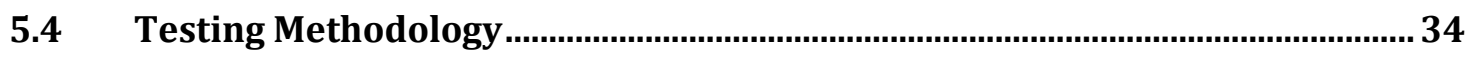

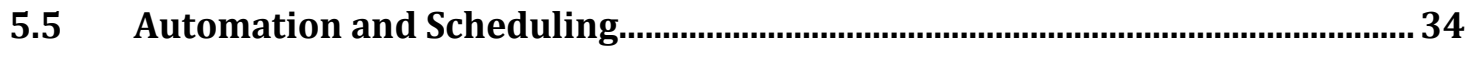

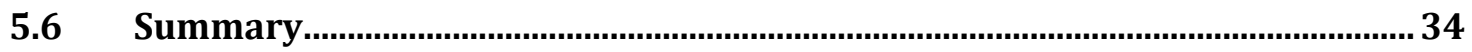

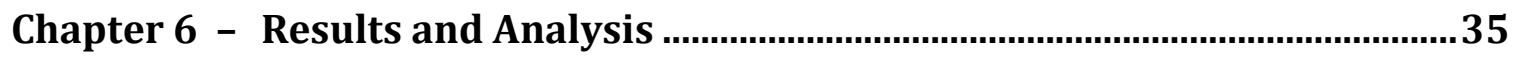

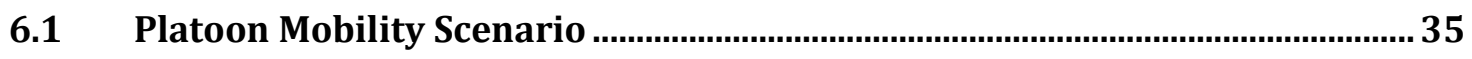

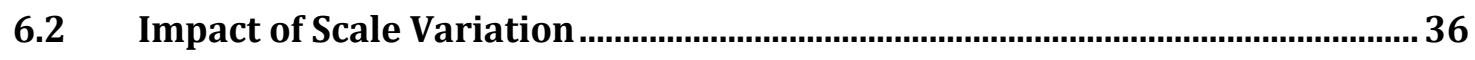

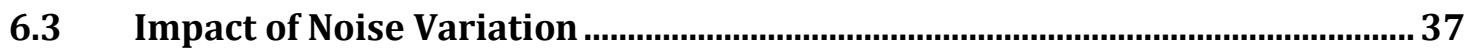




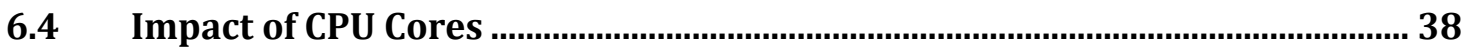

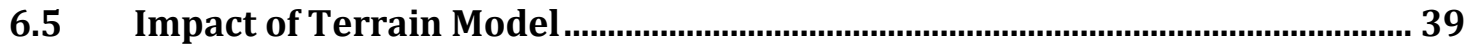

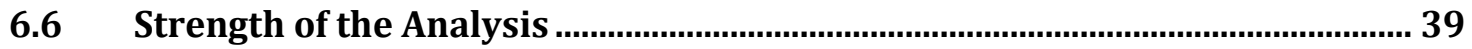

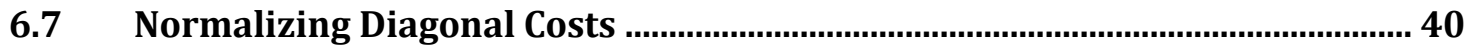

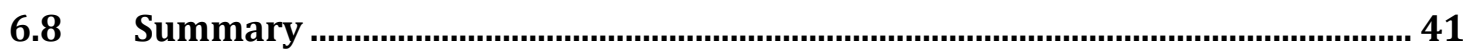

Chapter 7 - Conclusions and Future Work ..........................................................43

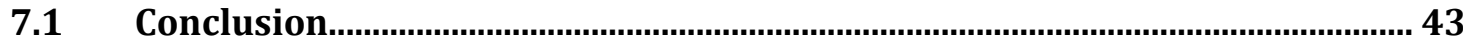

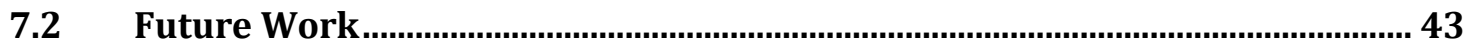

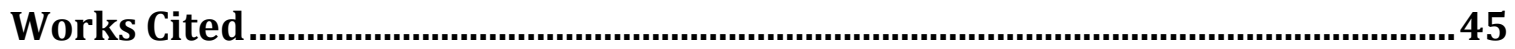

Appendix A. Analysis of the Yellowstone Dataset...........................................47

Appendix B. Analysis of the Yosemite Dataset.........................................................50 



\section{Table of Figures}

Figure 1-1: Yellowstone National Park, the development dataset............................ 2

Figure 1-2: Yosemite National Park, the testing dataset....................................... 3

Figure 1-3: Weighted sum of feature rasters for Yellowstone National Park............. 4

Figure 2-1: Voronoi diagram for a set of points .............................................. 7

Figure 2-2: Three resampling geometries, Rook, Queen and Knight ...................... 8

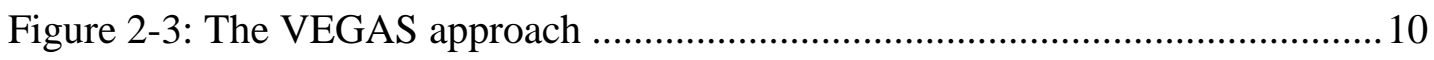

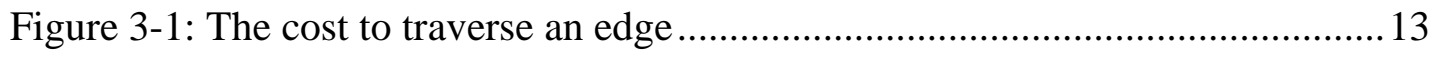

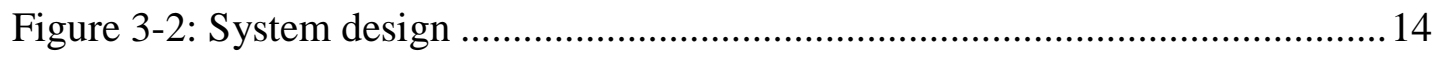

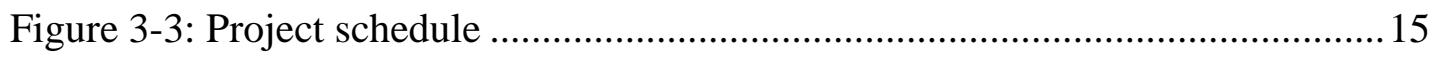

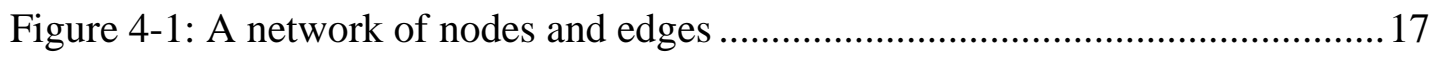

Figure 4-3: The template network dataset provided with the project ......................20

Figure 4-4: A weighted overlay of individual cost rasters ....................................22

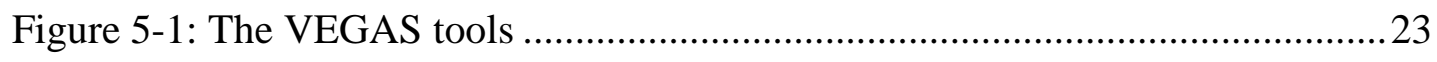

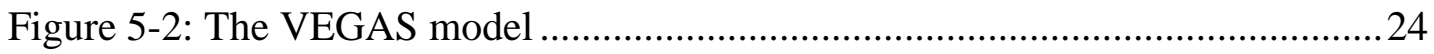

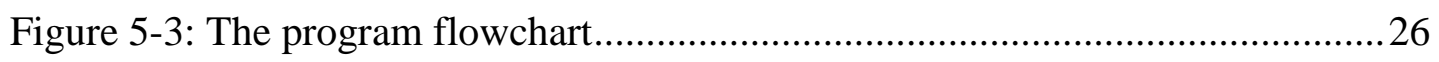

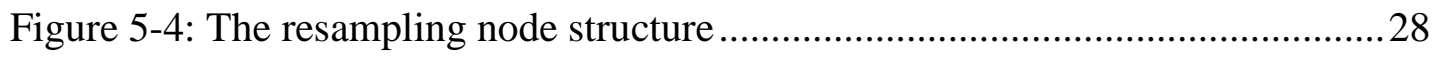

Figure 5-5: The vector model for queen geometry …........................................2 29

Figure 5-6: The creation of the template network dataset ................................... 30

Figure 5-7: Change in node values after running the Randomize tool ..................... 31

Figure 5-8: Multiple copies of the master database ............................................ 32

Figure 5-9: The CPU utilization during a simulation run with multi-processing .......33

Figure 5-10: Result of simulation depicting most frequently traversed edges ..........33 
Figure 6-1: Most frequently traversed edges between source and destination points36 Figure 6-2: A graph of scale vs. computation time …………………………….... 37

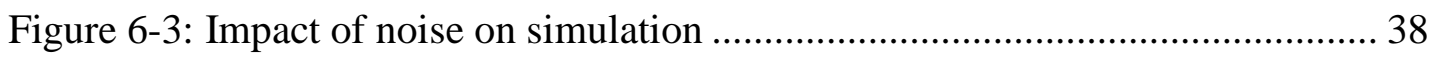

Figure 6-4: Impact of the number of cores on computation time............................... 39

Figure 6-5: Comparison of three simulation runs of the tool ..................................... 40

Figure 6-6: Comparing routes with un-normalized costs with normalized costs...... 41 


\section{List of Tables}

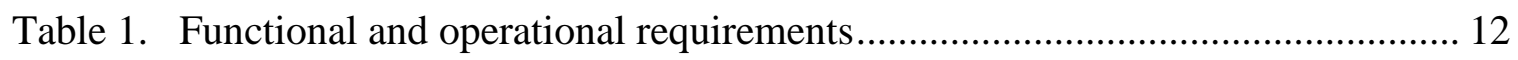

Table 2. The schema for the nodes feature class...............................23

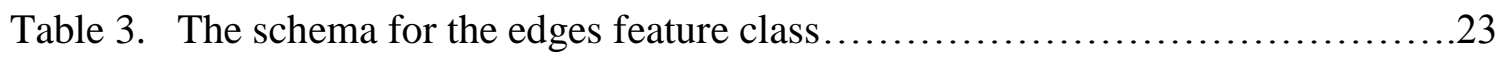

Table 4. The datasets compiled for each national park ................................26

Table 5. Effect of scale variations on accuracy and computation time.................47 



\section{List of Acronyms and Definitions}

Cost surface - a raster grid where the pixel values represent desirability of a factor influencing the terrain

VEGAS - Voronoi Diagram Extraction, Generalization and Simulation

API - Application Programming Interface

Network dataset - a data structure used by the ArcGIS Network Analyst extension to run route solvers

GUI - graphical user interface 



\section{Chapter 1 - Introduction}

When a large number of routes pass through a narrow space or a constricted region on a terrain they create bottlenecks which affect the flow of goods and people. The ability of such regions to influence and intercept many routes simultaneously makes them valuable in the study of human mobility. For example, while planning the mobility of an infantry platoon across a terrain, a GIS analyst could treat bottlenecks as possible ambush points and avoid paths that traverse through the constricted regions. While their importance is easy to comprehend, the process of finding such bottlenecks is not trivial. This paper describes the concept and implementation of one such method that uses route simulation to find bottlenecks on a terrain.

Section 1.1 introduces the client for the project. The problem is defined in Section 1.2. Section 1.3 presents the goals, objectives and scope of the proposed solution. Section 1.4 lists the target audience for the paper. An overview of the paper is presented in Section 1.5.

\subsection{Client}

Christopher Price, a GIS Analyst in the U.S. Government, was interested in modeling human mobility over a terrain for planning search and rescue and conflict avoidance operations. He applied various exploratory GIS tools to gain new insights into a terrain to predict optimal patterns (paths) for human mobility. One such suite of tools found bottlenecks on a terrain by using route simulation but required sophisticated hardware and software. Mr. Price believed that a portable version of the tools that could run on a laptop computer would make them more effective and accessible to a wider audience.

\subsection{Problem Statement}

In 2007, GIS analysts within the U.S. Government developed an algorithm to find bottlenecks on a terrain using route simulation. While their approach was innovative, their implementation of the algorithm was computationally intensive and required a farm of computers for processing. Only analysts with access to sophisticated hardware and software could utilize these tools making them impractical for use in the field.

\subsection{Proposed Solution}

The solution to finding the bottlenecks over a terrain had to address two main concerns:

1. Efficiency

2. Portability

The solution to the problem was to re-implement the original algorithm in a suite of Python tools using ArcMap and the ArcGIS Network Analyst extension. The solution addressed the concern of computational efficiency by using multiprocessing which allows the tools to run route simulations in multiple processes simultaneously, fully utilizing available hardware. The tools do not need any network connectivity and they rely solely 
on ArcGIS Desktop 10.1 and Python 2.7, making it possible to run them on a laptop computer in the field.

\subsubsection{Goals and Objectives}

The goal of the project was to highlight areas on a terrain that were most susceptible to congestion by simulating a large number of routes. In order to simulate routes efficiently, the first objective was to build a tool that resampled the input raster-based surfaces to a coarse grained vector network. This conversion from raster to vector was deemed necessary because the least-cost path route finding implementations, an essential component of the algorithm, were more efficient on a vector network than on rasters. Once the vector network was established, the next objective was to build another tool that could simulate routes between source and destination points by iteratively applying a noise value to the underlying cost model of the vector network. The final objective was to build a tool that could combine the simulated routes and plot them on a map to highlight the congested regions.

\subsubsection{Scope}

The project scope was divided into many sub parts. In order to build the tools a sample dataset was required. However, the client was unable to provide original data due to security restrictions. Therefore, two datasets, one for the Yellowstone National Park region (Figure 1-1) and another for the Yosemite National Park region (Figure 1-2) were assembled for development and testing purposes. During the development phase, the large problem was broken down into small geoprocessing tools, each performing a specific task. The tools were packaged into a toolbox and then tested on the two national park datasets. The final deliverables to the client included the source code for the tools, help documentation, training manuals, and the compiled datasets.

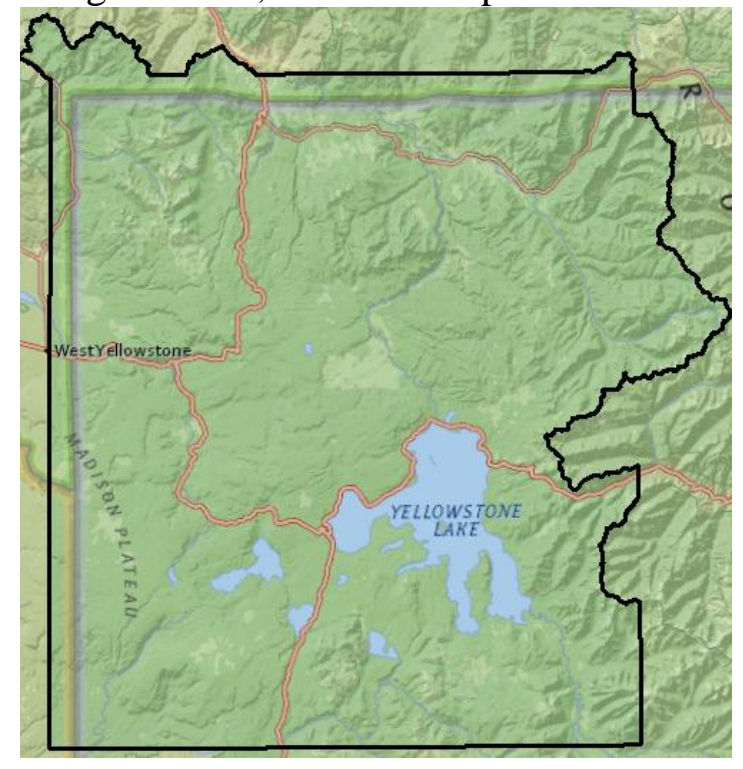

Figure 1-1: Yellowstone National Park, the development dataset 


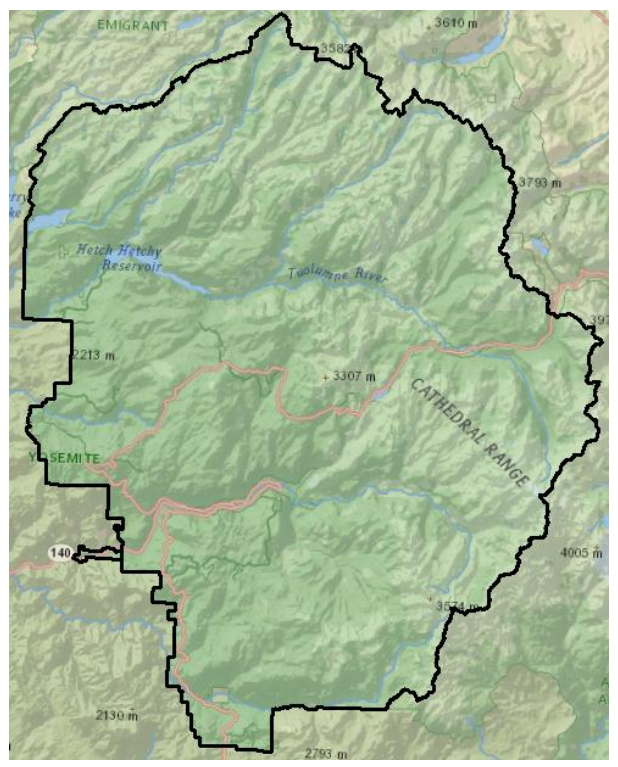

Figure 1-2: Yosemite National Park, the testing dataset

\subsubsection{Methods}

The first step in the project was gathering requirements and understanding the client's motivations. This was achieved through telephone and email conversations with the client. Though the client was unable to provide any formal documentation on the original approach, his detailed descriptions of the approach made it possible to design and develop the tools.

The client described a scenario in which an infantry platoon was to be mobilized across a terrain to reach a specific destination by avoiding enemy detection and ambush. Various terrain features like elevation, land use, roads, trails, observation posts were considered during the data collection phase. The terrains of the Yellowstone and Yosemite National Parks were considered for their ruggedness and the availability of comprehensive datasets. Individual datasets were scrubbed, loaded into a database, and then reclassified on a 0 to 100 scale where the 0 was the value assigned to the region that was most suitable for traversal. The reclassified rasters were then stacked and a weighted sum was computed to create a final cost raster surface as shown in Figure 1-3. 


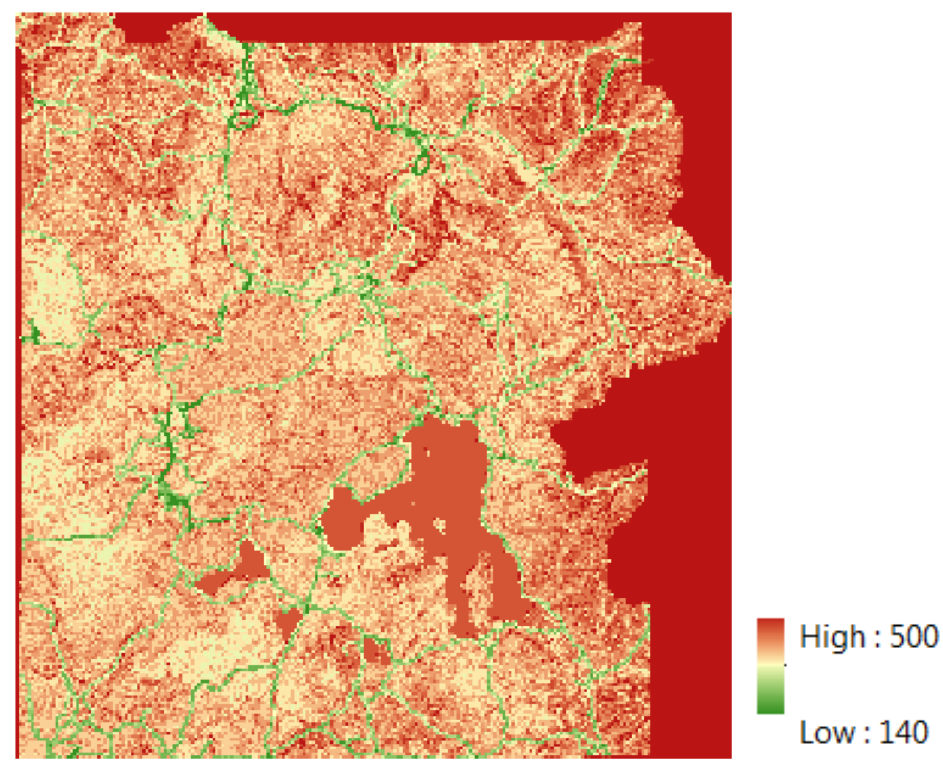

Figure 1-3: Weighted sum of feature rasters for Yellowstone National Park

The development process began by building a series of prototype tools for resampling the cost surface to a coarse grained vector network, creating the ArcGIS network dataset from the vector network, and simulating routes between source and destination points. The tools were tested on the Yosemite National Park dataset and presented to the client for approval. The client reviewed the tools and proposed some improvements which were incorporated during the development phase. The prototype tools were then converted to production code and documentation (help) strings were added to the tool. The final deliverable to the client included the compiled datasets, Python geoprocessing tools for ArcMap, source code, and a training manual.

\subsection{Audience}

This paper is geared towards students of GIS, professional analysts, and computer programmers who are well versed in GIS concepts and tools such as raster-based terrain models, least-cost paths, and simulation. GIS analysts in the fields of transportation, security, and defense may find the tool concept and implementation useful in their work and research. While readers who are familiar with the original approach will find the paper easy to follow, care has been taken to provide a clear and in-depth description of the new implementation.

\subsection{Overview of the Rest of this Report}

Chapter Two sets up the context and background research for the project. Chapter Three presents the overall system design. The database model employed by the project is described in Chapter Four. The heart of the project is the implementation, which is 
presented in Chapter Five. Chapter Six describes the results of the analysis tools. Finally, Chapter Seven presents the conclusion for the project and lists future scope. 


\section{Chapter 2 - Background and Literature Review}

The vector-based mobility modeling tools implement the Voronoi diagram Extraction, Generation and Simulation algorithm (VEGAS), developed by analysts in the U.S. government. While the algorithm's documentation is not available to the public, sufficient detail was provided by the client to reconstruct the original algorithm. To test the soundness of the VEGAS approach, the algorithm was broken down into its constituent elements, namely: Voronoi diagrams, least-cost path analysis, and Monte Carlo simulation.

Section 2.1 introduces the theory of Voronoi diagrams and describes some of their applications. Least-cost path analysis is described in Section 2.2. The statistical method of Monte Carlo simulation is described in Section 2.3. Section 2.4 summarizes the algorithm.

\subsection{Voronoi Diagrams}

Given a set of points in continuous space, a Voronoi diagram (Figure 2-1) divides the continuous space into a set of regions such that points within each region are closest to one of the known points.

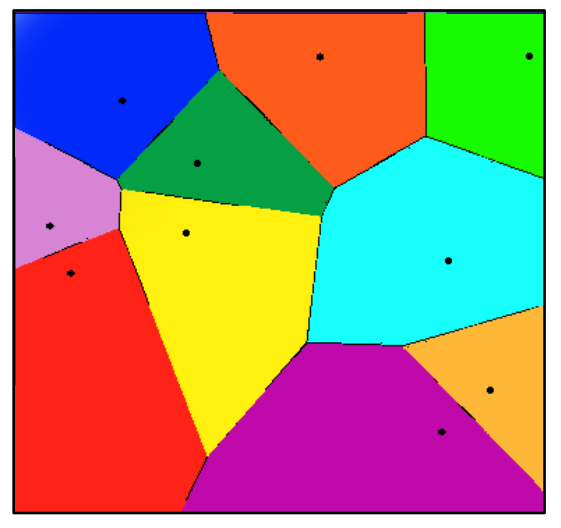

Figure 2-1: Voronoi diagram for a set of points

Voronoi diagrams (or Dirichlet regions or Thiessen's polygons) have found various applications from physics to astronomy and from meteorology (Thiessen \& Alter, 1911) to urban planning. A comprehensive study of Voronoi diagrams is described in Okabe, Boots, Sugihara, and Chiu (1992). The VEGAS algorithm applies Voronoi diagrams to generate paths on a terrain with each point in the set representing a road block or a nontraversable region. As a result, the input raster-based surface (terrain) could be converted to a network model for path simulation. This technique has been successfully implemented (with an algorithmic complexity of $\mathrm{O}(\mathrm{n} \log (\mathrm{n}))$ in various path planning operations in robotics (Bhattacharya \& Gavrilova, 2007). An improvement in the Voronoi diagram based path planning approach was described in Garrido, Moreno, Abderrahim, and Martin (2006) which applies a second step of 'Fast Marching' (Sethian, 1998) on the Voronoi diagram paths. 
The generation of Voronoi diagrams added complexity to the VEGAS algorithm and therefore a simpler approach was sought in the subsequent implementation of the algorithm. The modified approach relied on structured geometry to resample the rasterbased surface and generate a vector based network. The geometries of Rook, Queen and Knight were defined for this purpose (Figure 2-2).
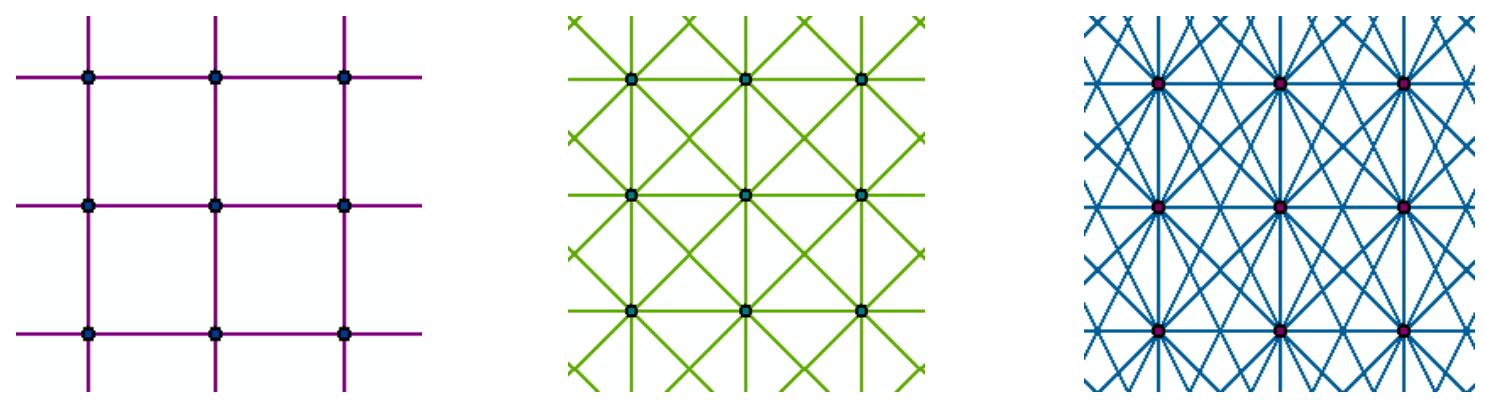

Figure 2-2: Three resampling geometries: Rook, Queen and Knight

While little information could be gathered as to how the structured geometries improved upon the Voronoi diagrams, interesting research in the field of Chess was found that described the effectiveness of each of the geometries on the chessboard, an 8 by 8 square grid. DelSanto (2011) in his presentation compared the effectiveness of the three chess pieces in covering the chessboard. He concluded that "... it is impossible to checkmate an opponent with solely a bishop and king, or solely a knight and king. It is possible to checkmate an opponent with just a rook and a king (and therefore obviously a queen and a king)" (pg 8). Applying the same principles to the network connectivity model, it was clear that while knight geometry provided the most coverage, it was also the most complex to compute. On the other hand, the rook geometry provided the coarsest coverage but it was very efficient in terms of path computation.

\subsection{Least-Cost Path Analysis}

Throughout history humans have travelled between points following a shortest path (Murrieta-Flores, 2010). A shortest path attempts to optimize one or more attributes like travel time, distance, effort or security. Dikjstra (1959) presented an algorithm for computing the shortest (least-cost) path between two nodes in a graph. This algorithm, along with its derivatives, has found a tremendous number of applications in the fields of mathematics, engineering, and geographical sciences. Most geographical information systems implement some version of the least-cost path algorithm for solving optimal path finding and resource allocation problems.

According to Bagli, Geneletti, \& Orsi (2011), "Least-cost path analysis (LCPA) allows designers to find the cheapest way to connect two locations within a cost surface, which can be computed by combining multiple criteria, and therefore by accounting for different issues (environmental impact, economic investment, etc.)" (pg 235). The authors proposed an optimal path for routing power lines using multi-criteria evaluation (MCE) and LCPA. This approach forms the basis of the VEGAS algorithm to highlight areas of the terrain susceptible to congestion and bottlenecks. In order to study the impact 
of power lines, Bagli and others created a raster-based cost surface composed of factors affecting human health, landscape and nature such as density of buildings, distance from buildings, average height of buildings, distance from highly valued cultural and recreational sites, aspect, etc. High costs were assigned to regions of the terrain which demonstrated factors that were detrimental to the presence of power lines. LCPA was then applied from the source to destination points and a path was produced that was the least destructive (intrusive) to its surroundings. In the VEGAS approach, factors influencing the mobility of humans, such as aspect, land use, presence of roads and obstructions were considered for cost evaluation. These factors were then combined into a cost raster and provided as input to an LCPA. The LCPA outputs a path that was most optimal for a human to traverse on the cost surface.

Relying only on LCPA to compute the most optimal path had a drawback. It did not take into account any uncertainty (variation) in the cost surface computation. Therefore the VEGAS approach added a third step to its analysis, that of Monte Carlo simulation.

\subsection{Monte Carlo Simulation}

Geographic measurements such as distances, elevations, land use classifications, delineations of features from satellite images, GPS positions, etc. are rarely very precise and often introduce an uncertainty in decision making. The Monte Carlo simulation technique addresses this uncertainty in measurement by considering a large number of random samples and quantifying the uncertainty on a statistical scale to allow for better decision making. Zhou et al. (2003) applied this technique to create a landslide hazard probability map in Japan by analysing the influence of slope and groundwater factors on slope failures through simulation. Jahanpeyma, Delavar, Malek, \& Kamalian (2011) used Monte Carlo simulations to measure the propogation of error while quantifying the siesmic vulnerability of the city of Tehran, Iran. They concluded that "the variation of variances of seismic vulnerability assessment obtained in this research is inversely related the number of repetitions in Monte Carlo simulation" (pg 5) indicating the importance of simulations to rule out uncertainity and take measureable risk.

The VEGAS approach relied on simulation to account for uncertainty in its multicriteria evaluation of the cost surface. Each iteration of the simulation changed the underlying cost model by $5-10 \%$ and generated a new path between source and destination points by running LCPA. The large numbers of simulated routes accounted for optimal and sub-optimal paths between points, highlighting potential bottlenecks.

\subsection{Summary}

The VEGAS algorithm has used an innovative combination of established techniques like multi-criteria evaluation, LCPA and Monte Carlo simulation (Figure 2-3) to produce a large number of routes to highlight regions susceptible to bottlenecks.

While Chapter 2 has focused on the VEGAS algorithm, the next few chapters discuss the new implementation of the algorithm in substantial detail. 


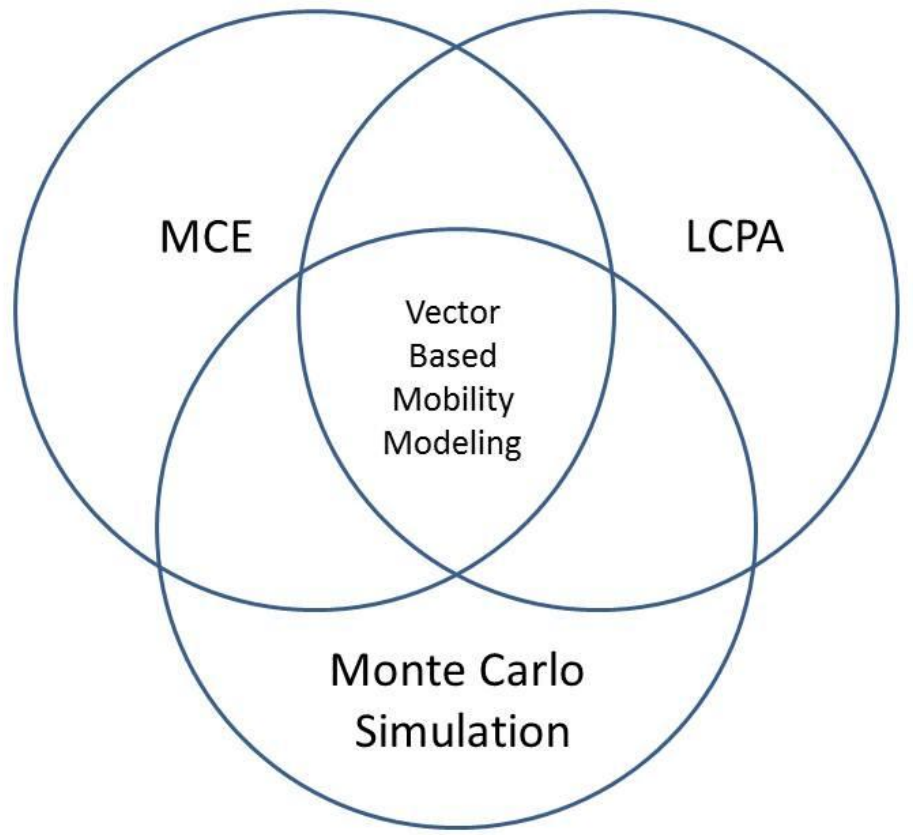

Figure 2-3: The VEGAS approach 


\section{Chapter 3 - Systems Analysis and Design}

System analysis and design are the most important phases in the project lifecycle and have a tremendous influence on the project outcome. This chapter begins by revisiting the problem of computing bottlenecks on a terrain in Section 3.1. The functional and the operational requirements that were developed in collaboration with the client are presented in Section 3.2. Section 3.3 details the overall system design. The project plan and schedule are discussed in Section 3.4. Section 3.5 presents the chapter summary.

\subsection{Problem Statement}

Analysts within the U.S. Government developed a strategy called VEGAS to highlight regions on a terrain that could become traversal bottlenecks or hotspots. The ability of such regions to intercept and influence many traversing paths simultaneously makes them vital in the modeling of human mobility. Such bottlenecks play an important role in a wide variety of applications ranging from rescue mission planning to security and defense operations.

However, the original implementation of the VEGAS approach relied on a complex set of software packages including Java and Matlab and needed the computing power of a farm of computers to perform large numbers of simulations. As a result, the implementation was not portable and could not be taken into the field. Only analysts with access to sophisticated hardware and software could utilize the implementation, limiting its potential. With advances in GIS technology, it was felt that the method could be reimplemented in a much more efficient manner that did not need sophisticated hardware or software.

\subsection{Requirements Analysis}

The process of system design began by understanding the context and the motivations of the problem. This was achieved by engaging the client in a conversation by telephone and email. The client presented the background for the project through a set of PowerPoint slides that briefly touched on the VEGAS concept and highlighted some of the deficiencies in its implementation. While some general guidelines for the project were shared through a Word document, the security restrictions prevented the client from sharing the blueprints of the original implementation. This added a level of complexity to the project, as the algorithm had to be implemented from scratch.

The algorithm and the specification for the project were developed iteratively under the client's guidance and technical direction. A substantial amount of time was spent understanding the client's use cases. Background research and literature review of the different methods applied by the algorithm filled in the missing pieces of the puzzle.

While communicating with the client it was clear that client's limited availability was going to pose a challenge. Thus it became imperative that all the requirements had to be gathered up front. In order to mitigate risks it was decided that the project should build rapid prototypes early on and get a buy off from the client. Table 1 organizes the various requirements developed as a part of the project specification into functional and 
operational requirements. While the operational requirements governed the runtime environment, the functional requirements were the fundamental goals that the project had to achieve success.

Table 1. Functional and operational requirements

\begin{tabular}{|c|c|c|}
\hline $\begin{array}{l}\text { Requirement } \\
\text { Type }\end{array}$ & Requirement & Description \\
\hline \multirow[t]{6}{*}{ Functional } & Multiple input rasters & $\begin{array}{l}\text { The tools had to accept as input, one or } \\
\text { more raster-based factors like slope or } \\
\text { land-use that influenced human traversal. }\end{array}$ \\
\hline & Vector resampling & $\begin{array}{l}\text { To speed up the least-cost path analysis, } \\
\text { the raster surfaces needed to be } \\
\text { resampled to a coarse grained vector grid }\end{array}$ \\
\hline & $\begin{array}{l}\text { Rook, queen and knight } \\
\text { resampling geometries }\end{array}$ & $\begin{array}{l}\text { The resampling process had to follow a } \\
\text { structured geometric pattern. }\end{array}$ \\
\hline & Least-cost path & $\begin{array}{l}\text { In order to highlight bottlenecks, a large } \\
\text { number of least-cost path routes had to be } \\
\text { computed between the given source and } \\
\text { destination points. }\end{array}$ \\
\hline & Incorporate uncertainty & $\begin{array}{l}\text { Each iteration of the simulation had to } \\
\text { apply a noise value to alter the } \\
\text { underlying cost model to generate a new } \\
\text { least-cost path. }\end{array}$ \\
\hline & $\begin{array}{l}\text { Python based ArcMap } \\
\text { tools }\end{array}$ & $\begin{array}{l}\text { The tools were to be developed in Python } \\
\text { using ArcMap API. }\end{array}$ \\
\hline \multirow[t]{2}{*}{ Operational } & $\begin{array}{l}\text { Efficient use of } \\
\text { available hardware }\end{array}$ & $\begin{array}{l}\text { The tools had to use multi-processing to } \\
\text { improve the efficiency by running } \\
\text { simulations in parallel, independent } \\
\text { processes on the same computer. }\end{array}$ \\
\hline & Training manual & $\begin{array}{l}\text { Help documentation and a training } \\
\text { manual had to be provided to get the } \\
\text { client up to speed with the } \\
\text { implementation. }\end{array}$ \\
\hline
\end{tabular}




\subsection{System Design}

The client's expertise in esri's ArcGIS technology directed the implementation towards a suite of Python tools for ArcMap. Early prototypes using ArcGIS Network Analyst and Python's multi-process capabilities paved the way for the overall system design that is presented in this section. The system design that evolved from prototypes to meet the requirement consisted of following four sub-systems:

\subsubsection{Input and User Experience}

The input and user experience sub-system was responsible for accepting and validating user input. This was achieved by leveraging ArcMap's ability to provide a graphical user interface (GUI) for geoprocessing tools written in Python. The GUI provided the user with the ability to browse and select one or more raster datasets, specify numerical values for the number of simulations and uncertainty, and select the resampling geometry from a drop down list. The GUI validated the user input based on pre-defined rules and it also allowed the user to visualize the tools through the ModelBuilder interface.

\subsubsection{Pre-processing}

The pre-processing sub-system consisted of a set of two tools that resampled the input rasters based on the user defined geometry to produce a coarse grained network dataset. This pre-processing step was required because it gave the user the ability to change the resolution of the analysis instead of the fixed resolution of the raster. The process of resampling performed an overlay sum of all the pixels for the input rasters for a selected row and column. The cost of traversing an edge (Figure 3-1) was computed as the difference in the values of the adjacent pixels. This led to the creation of a network dataset consisting of nodes and edges which became the input to the simulation module.

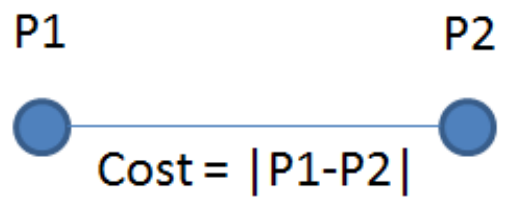

\section{Figure 3-1: The cost to traverse an edge}

\subsubsection{Simulation}

The simulation module was responsible for running the least-cost path analysis repeatedly to generate a large number of routes between the source and destination points. For each simulation to yield a new route between the points, the resampled cost surface was altered by varying the cost of each edge by a user defined amount. This accounted for uncertainty and inaccuracy in the measurement and classification of the original cost surface. The simulation module was implemented in a set of 2 tools that applied multi-processing to run the simulations in multiple processes simultaneously. 


\subsubsection{Output and Analysis}

The simulation tools kept an account of every generated route in a database. They tracked the number of times each edge was traversed in the vector network. At the end of the simulation, the master process gathered the simulation result from each of its multiple processes and copied them into a master database. Once sufficient numbers of routes were generated, the output module computed the frequency of each edge in the list of simulated routes and plotted them on a map to highlight those edges and nodes that were most traversed. The overall system design is shown in Figure 3-2.

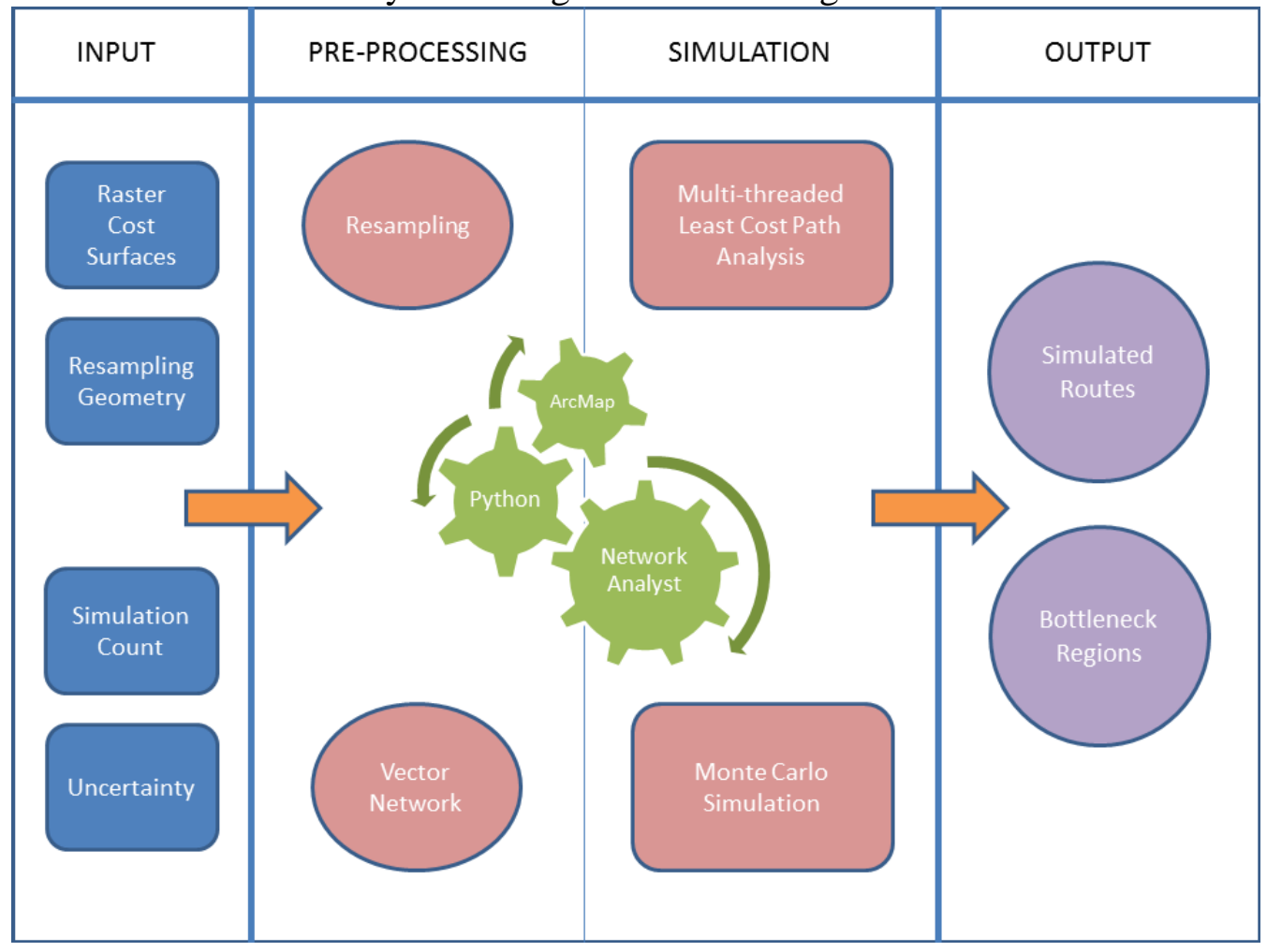

Figure 3-2: System design

\subsection{Project Plan}

The classic waterfall model was adopted for the project, considering the client's limited availability and the project having most of its requirements and specifications collected at the beginning of the project. The project was organized into 11 phases from requirement analysis to final delivery. Each phase had a defined timeline and quantifiable deliverables to measure its progress. The project constantly strove to minimize and mitigate risks at each phase. As the end date for the project was fixed (December 2013), a schedule (Figure 3-3) was created by walking backwards from the end date. 


\begin{tabular}{|l|l|l|l|l|l|l|l|l|l|l|l|l||}
\hline & JAN & FEB & MAR & APR & MAY & JUN & JUL & AUG & SEP & OCT & NOV & DEC \\
\hline Requirements & & & & & & & & & & & & \\
\hline Proposal & & & & & & & & & & & & \\
\hline Plan & & & & & & & & & & & & \\
\hline Design & & & & & & & & & & & & \\
\hline Prototype & & & & & & & & & & & & \\
\hline Develop & & & & & & & & & & & & \\
\hline Test & & & & & & & & & & & & \\
\hline Document & & & & & & & & & & & & \\
\hline Defend & & & & & & & & & & & & \\
\hline Final review & & & & & & & & & & & & \\
\hline Delivery & & & & & & & & & & & & \\
\hline
\end{tabular}

\section{Figure 3-3: Project schedule}

The first four phases of the project from requirements analysis to design focused on understanding the project goals and creating a blueprint for the project. The progress during this stage was slow, as pieces of the puzzle had to be gathered and put together to form a coherent picture. This stage also doubled as a data collection phase because data could not be provided by the client due to security restrictions. Once the design was ready, the next goal was to acquire relevant technical skills needed to complete the project. As the project had to implement the algorithm from ground up, substantial amount of time was allotted for the prototype and development phases. This decision proved to be beneficial during the project lifecycle. Risks associated with relatively unknown areas of the system design such as the Python-based network routing libraries, multi-processing, and vector resampling were mitigated early on in the prototype phase. The prototypes were demonstrated to the client in July. With the client's approval, the development phase converted the prototype code into final tools. During the testing phase that followed, software issues were found and fixed in the implementation. The tools were annotated with help strings and a help manual created for the client in the documentation phase. The final project implementations of the tools, source code were delivered to the client along with compiled datasets and training manual in early October. This marked the successful completion of the project.

\subsection{Summary}

Without a strong system design and careful consideration, the goals and objectives of the project would not have been delivered on time. The next chapter on database design dives into the framework that supports the implementation. A detailed walk through of the project implementation is presented in Chapter 5. 


\section{Chapter 4 - Database Design}

The simulation tools developed in this project used a geodatabase to store the input data, execution state, intermediate results, and the final output. This chapter introduces the database design for the simulation tools. Section 4.1 presents the conceptual data model. The database schema and the logical model are presented in Section 4.2. During the project development, two datasets were compiled for development and testing. The source of those datasets and the collection method is presented in Section 4.3 and Section 4.4 respectively. The various pre-processing steps applied to the data are described in Section 4.5. Section 4.6 presents the chapter summary.

\subsection{Conceptual Data Model}

The analysis tools centered on the concept of a network, consisting of nodes connected to one or more edges, as shown in Figure 4-1.

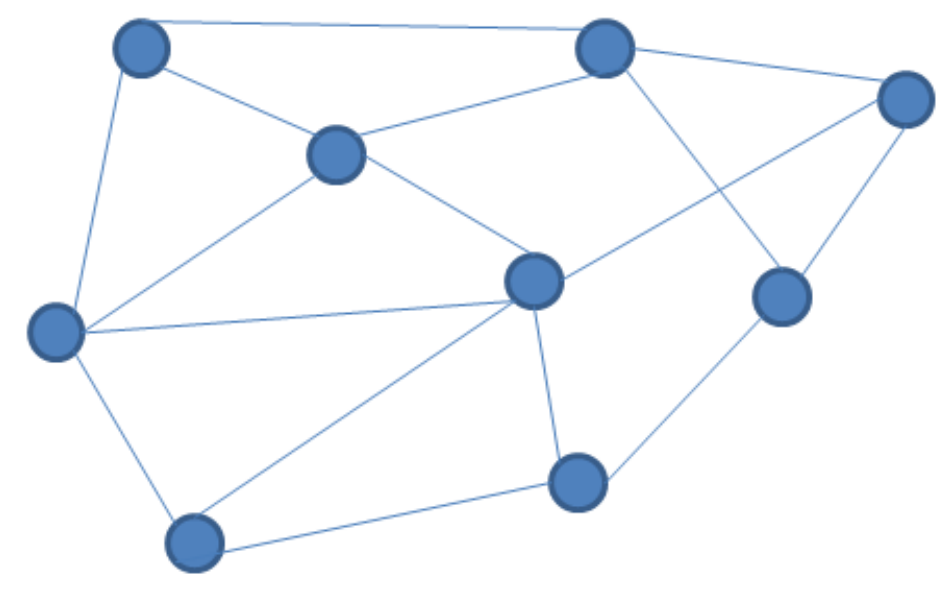

Figure 4-1: A network of nodes and edges

The network was derived from a raster surface by resampling the raster to a coarse grained grid. Each sampling point became a node in the network with value of the sampled pixel being assigned to the node. The cost to traverse an edge was computed as the difference in the values of the adjacent nodes. The tools performed a least-cost path analysis on the network model between source and destination points that resulted in a route which was made up of one or more edges. This relationship is depicted in Figure 42. 


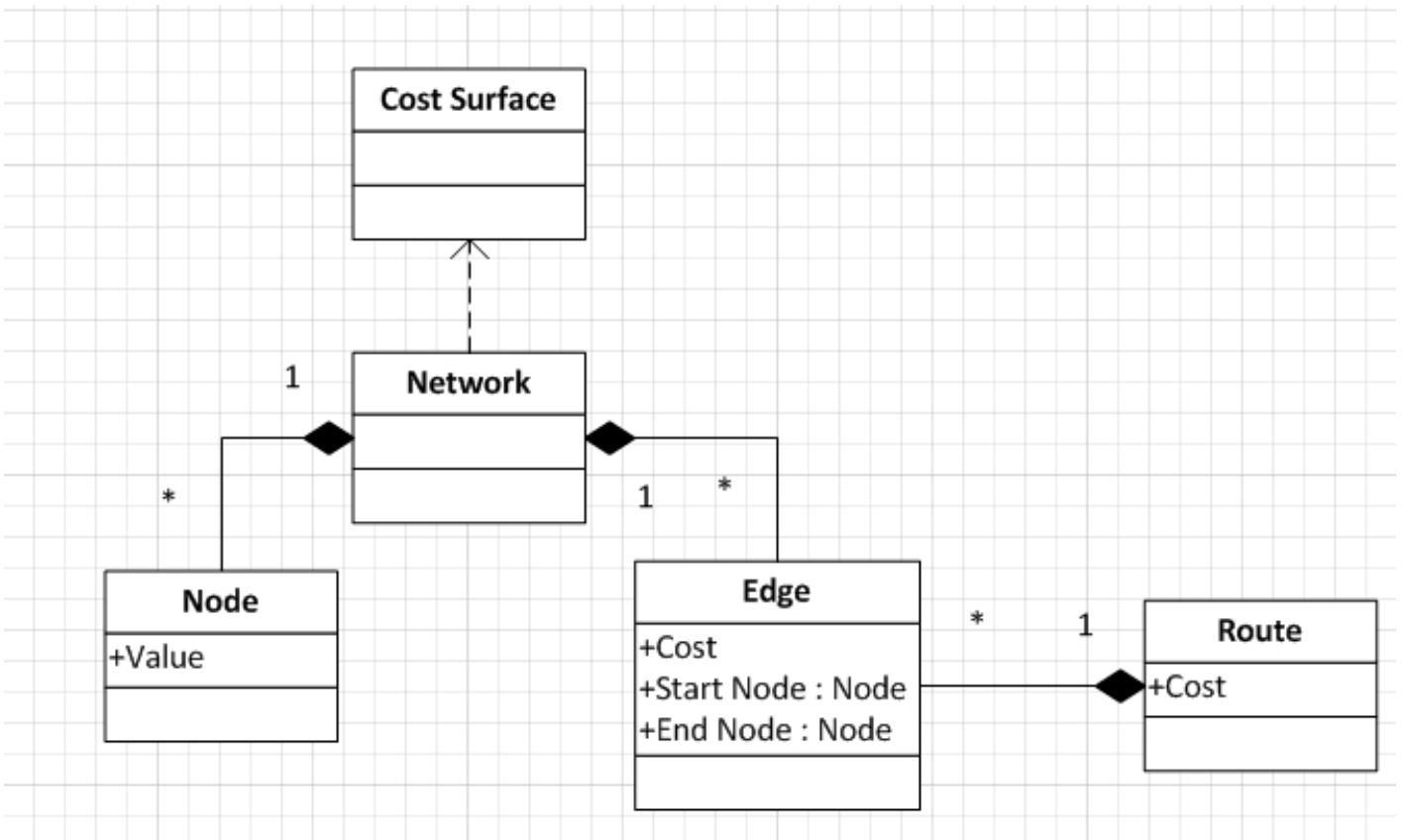

Figure 4-2: The network model

The analysis tools developed in this project generated a large number of routes and then plotted them on a map to highlight bottlenecks, which are regions where two or more routes pass through.

\subsection{Logical Data Model}

The input to the tools consisted of one or more raster files that modeled the cost surface for the terrain. Once the rasters are read, the process of resampling begins by creating two features classes to store the resampled nodes and edges. The schema of these feature classes is depicted in Table 2 and 3.

Table 2. The schema for the nodes feature class

\begin{tabular}{|l|l|l|}
\hline Field name & Data type & Purpose \\
\hline OBJECTID* & Object ID & A unique identifier for the row \\
\hline SHAPE* & Geometry & Stores the coordinates of the point \\
\hline ID & Long & A unique identifier for the point \\
\hline VALUE & Float & $\begin{array}{l}\text { Stores the sampled value of the raster } \\
\text { pixel }\end{array}$ \\
\hline
\end{tabular}


Table 3. The schema for the edges feature class

\begin{tabular}{|l|l|l|}
\hline Field name & Data type & Purpose \\
\hline OBJECTID* & Object ID & A unique identifier for the row \\
\hline SHAPE* & Geometry & $\begin{array}{l}\text { Stores the points that make up the } \\
\text { edge }\end{array}$ \\
\hline SRC_ID & Long & $\begin{array}{l}\text { The identifier of the starting node of } \\
\text { the edge (as defined in the nodes } \\
\text { feature class) }\end{array}$ \\
\hline DES_ID & Long & $\begin{array}{l}\text { The identifier of the ending node of } \\
\text { the edge }\end{array}$ \\
\hline COST & $\begin{array}{l}\text { The cost to traverse the edge from } \\
\text { source to destination }\end{array}$ \\
\hline REPEATED & Long & $\begin{array}{l}\text { The number of times an edge was } \\
\text { repeated in all the simulated routes }\end{array}$ \\
\hline SHAPE_Length* & Double & $\begin{array}{l}\text { The length of the edge as computed } \\
\text { by Geodatabase }\end{array}$ \\
\hline
\end{tabular}

The nodes feature class was populated with rows which were sampled at regular intervals based on the input resolution. The VALUE attribute of each row was assigned the overlay sum of the pixel values of all the input rasters for a specific row and column. The edges in the edges feature class were then created based on the input geometries Rook, Queen or Knight. The COST attribute was assigned the difference in the values of the nodes adjoining the edge. While this added redundancy, it made the least-cost path analysis run faster as the cost to traverse the edge was readily available. Each edge also maintained back links to the nodes that were used to create the edge. This was useful while adding uncertainty to the VALUE attribute of each node. The back links made it possible to efficiently update the cost of traversing the edge that joined two nodes.

In order to run least-cost path analysis using ArcGIS Network Analyst, the feature classes had be loaded into a network dataset. However, in the 10.1 version of ArcGIS, there was no convenient way to create a network dataset using the arcpy Python library. A pre-created template network dataset containing just the schema for the tables was provided with the project implementation as shown in Figure 4-3. After the nodes and feature classes were created, their data were loaded into the template network dataset. While simulating routes, each executing process made a copy of this network dataset. This extra copy was required because the 10.1 version of the file geodatabase did not support simultaneous write operations by multiple processes. Once the simulations were complete, all the generated paths from the Routes feature class were copied to the master database for further analysis. 


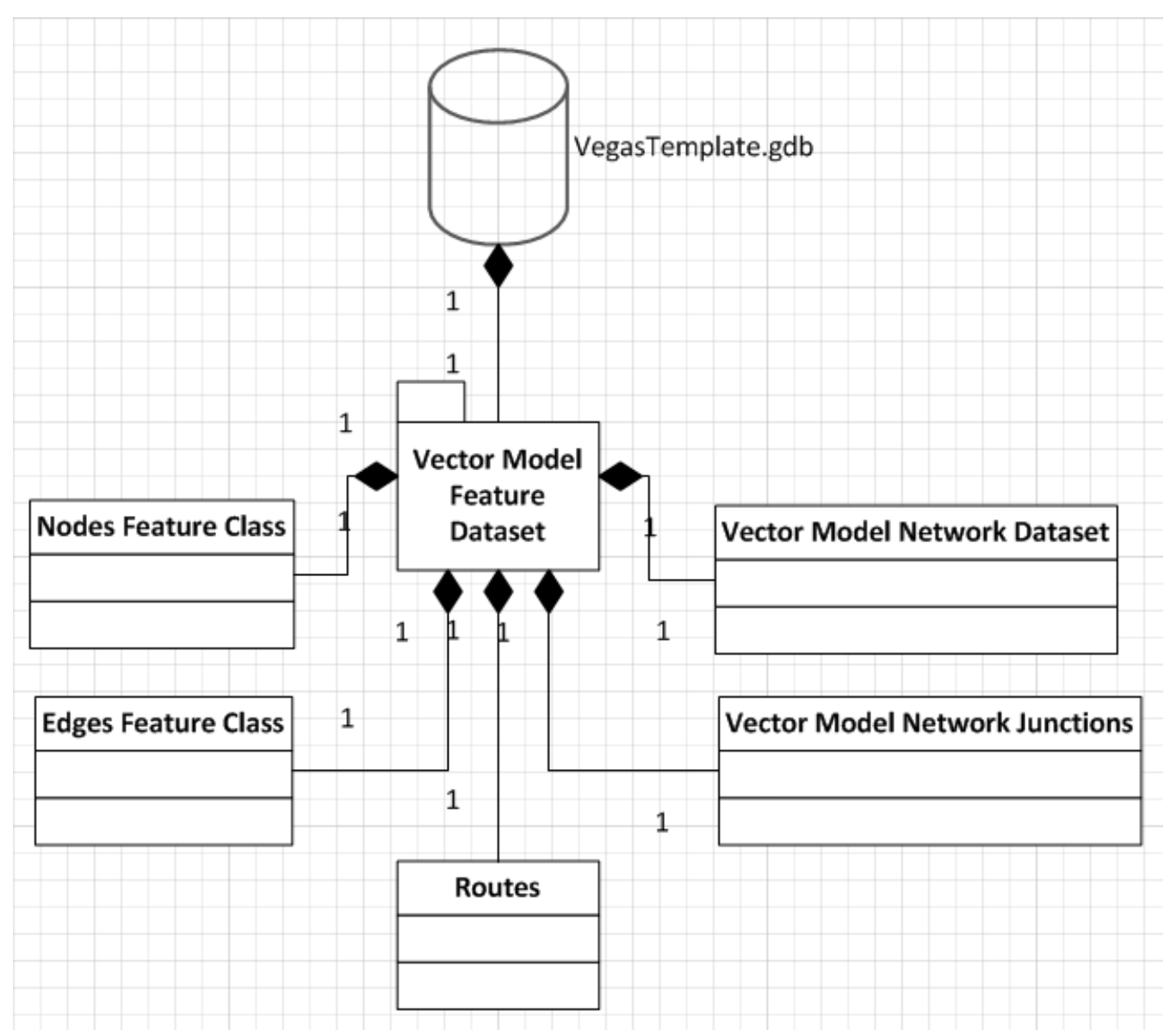

Figure 4-3: The template network dataset provided with the project

\subsection{Data Sources}

Two digital datasets, one for the Yellowstone National Park and the other for the Yosemite National Park, were gathered for planning the traversal of an infantry platoon across a terrain. The objective was to find optimal routes for the platoon to move across the terrain to their destination avoiding enemy detection and ambush. The Yellowstone dataset was primary used during the development of the tools, while the Yosemite dataset was primarily used for testing. The sources of the datasets are listed in Table 4. 
Table 4. The datasets compiled for each national park

\begin{tabular}{|l|l|l|}
\hline Name & Type & Source \\
\hline $\begin{array}{l}\text { Digital Elevation Model } \\
\text { (DEM) }\end{array}$ & Raster & $\begin{array}{l}\text { USGS Seamless Viewer } \\
\text { portal }\end{array}$ \\
\hline Land use & Raster & $\begin{array}{l}\text { USGS National Land } \\
\text { Cover Dataset (2006) }\end{array}$ \\
\hline Roads and highways & Vector & $\begin{array}{l}\text { National Park Service } \\
\text { (web) }\end{array}$ \\
\hline Park boundary & Vector & $\begin{array}{l}\text { National Park Service } \\
\text { (web) }\end{array}$ \\
\hline Park trails & Vector & $\begin{array}{l}\text { National Park Service } \\
\text { (web) }\end{array}$ \\
\hline Outposts & Vector & $\begin{array}{l}\text { National Park Service } \\
\text { (web) }\end{array}$ \\
\hline
\end{tabular}

\subsubsection{Data Collection Rationale}

The two national parks were considered for the problem because they resembled the rugged terrain of a combat front. To plan the traversal routes for a platoon, it was important to find areas of the terrain that were susceptible to enemy detection and ambush while considering their efficiency for the movement of soldiers and their equipment. Factors influencing the traversal such as slope of the terrain, land use, presence of roads and highways, trails and outposts were considered. Regions of the terrain with a gentle slope were more suitable for human traversal and the carrying of equipment. Therefore, when the cost surface was generated from the DEM, flatter regions or regions of gentle slope were assigned low costs. The land use dataset was used to create another cost surface where easily traversable regions such as flatlands, meadows, areas with small shrubs and trees were assigned lower costs as compared to areas of dense forests and water bodies like lakes and rivers. To create a cost surface from roads, highway and off-road trails vector files, the datasets were first rasterized and then reclassified by assigning lower costs to roads and highway regions based on the assumptions that a platoon can move faster on a paved road than on unpaved terrain. After all the raster surfaces were created, they were combined using a weighted overlay operation to output a final cost surface raster, as shown in Figure 4-4. 


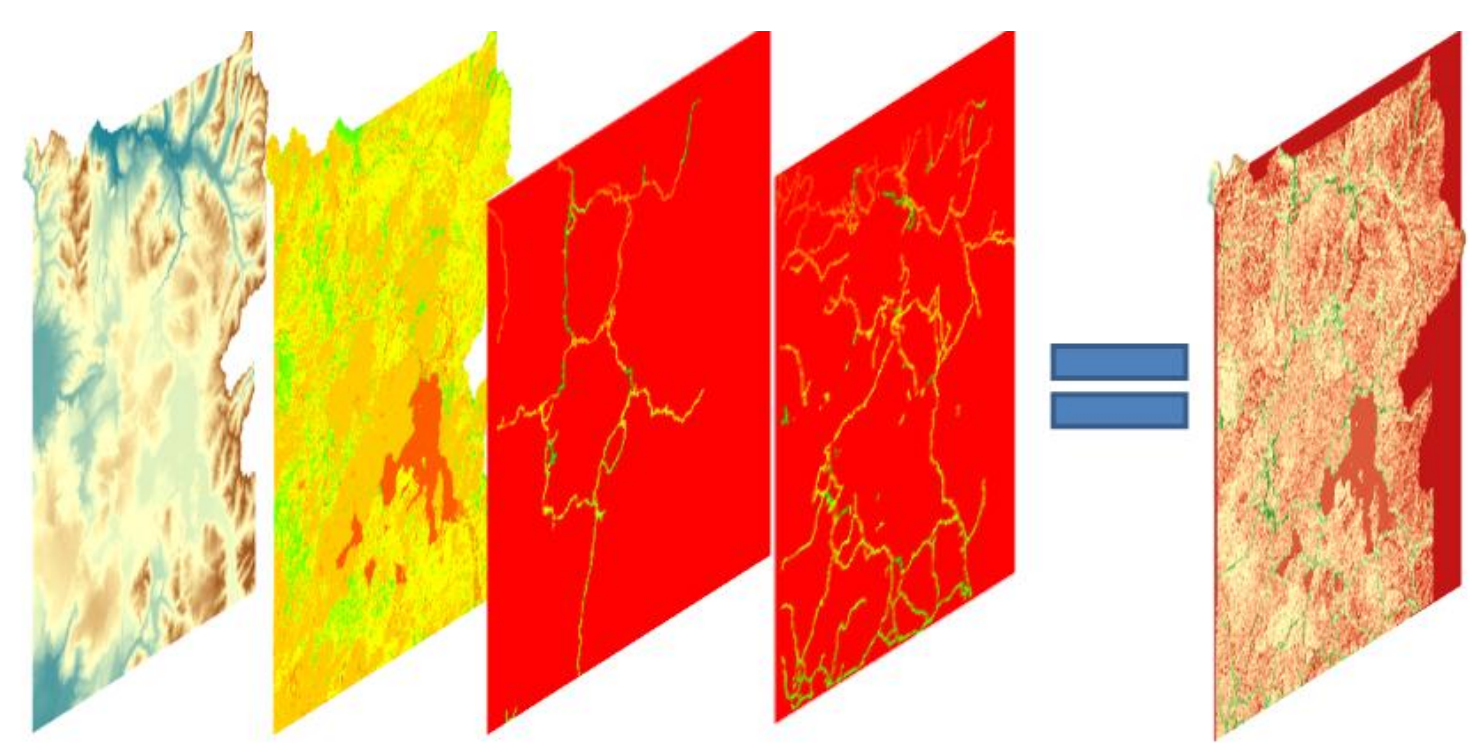

Figure 4-4: A weighted overlay of individual cost rasters

\subsection{Data Collection Methods}

Most of the project's data were digital and downloaded from the USGS and NPS web sites in raster and vector format for the Yellowstone and Yosemite national park regions. No physical data collection methods were applied to gather data.

\subsection{Data Scrubbing and Loading}

The raster and the vector datasets downloaded from the USGS and NPS web sites were loaded into a file Geodatabase as raster datasets and feature classes. To work within the study area, the datasets were clipped to the boundaries of the two national parks using the Clip tool in ArcMap. The vector datasets were converted to a raster format using the Vector to Raster tool. All the datasets were projected to their appropriate UTM projection zones: Zone 12 North for Yellowstone National Park and Zone 11 North for Yosemite National Park.

\subsection{Summary}

This chapter described the database schema used by the tools for simulating routes. It also touched upon the steps behind collecting the datasets to develop and test these tools. The next chapter provides a detailed view into the tool implementation and describes how the tools processed the data to simulate routes. 


\section{Chapter 5 - Implementation}

Chapter 5 presents the heart of this paper - the implementation. This chapter delves into the project implementation in a top-down manner starting with user experience in Section 5.1. Section 5.2 presents the implementation model. The Python tools that make up the implementation are described in detail in Section 5.3. Section 5.4 outlines the methodology used for testing the tools. Section 5.5 touches upon the automation and scheduling aspects of the tools. A summary of the implementation is presented in Section 5.6 .

\subsection{User Experience}

The Python-based geoprocessing tools developed in this project can be used in a standalone fashion (Figure 5-1) or as a complete model through the Model Builder interface within ArcMap.

\begin{tabular}{|c|}
\hline 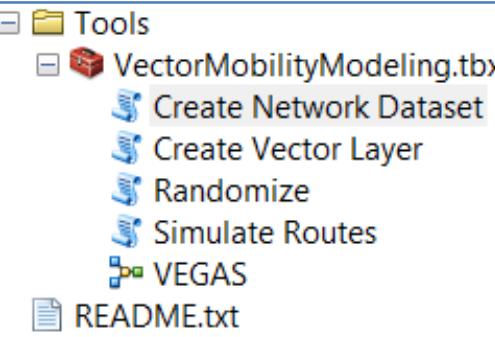 \\
\hline
\end{tabular}

\section{Figure 5-1: The VEGAS tools}

The Model Builder interface allows users to define program input, control fine grained parameters, and observe the program execution in a single graphical application. Once all the fine-grained parameters and defaults are configured, users can simply execute the model like any other geoprocessing tool. Figure 5-2 shows the screenshot of the model's GUI. 


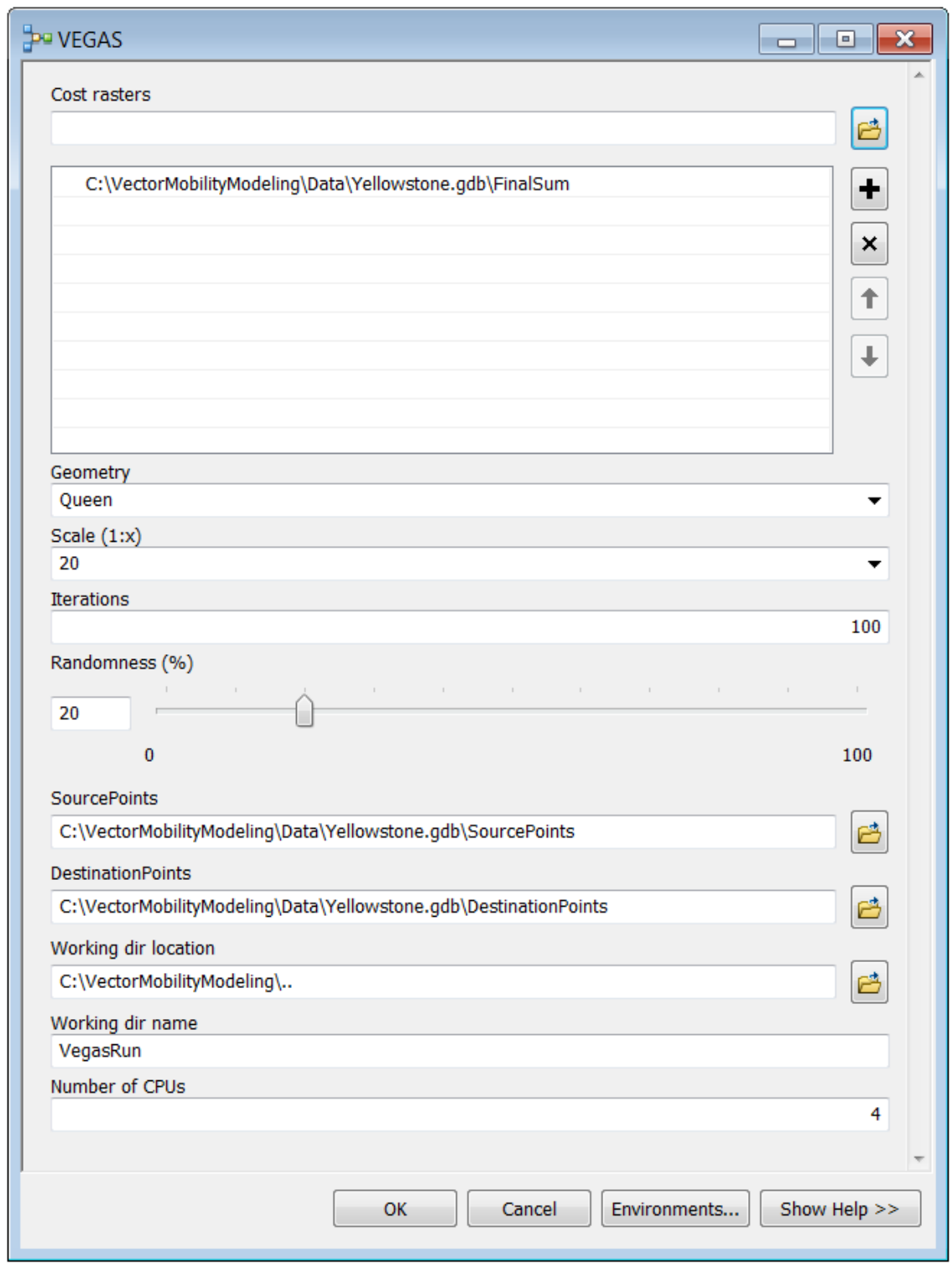

\section{Figure 5-2: The VEGAS model}

The model accepts as input one or more raster images that describe the terrain as a cost surface. The geometry parameter defines the resampling pattern when the rasterbased cost surfaces are converted to a vector network. Three input geometries are supported, that of Rook, Queen and Knight, based on increasing order of connectivity. The resolution of the resampling process is controlled by the scale parameter. Its value determines the complexity of the vector network and influences the time taken to complete the simulation. The randomness parameter defines the percentage of noise that must be added to the cost model while simulating routes to account for any uncertainty in the measurement of the cost surfaces. The source and destination points set the start and end points for route simulation. The working directory name and location define a file 
system folder where temporary data are created. The number of CPUs defines the degree of parallelism for simulation; typically it should be equal to the number of CPU cores on the computer running the tools. Once the parameters are input and the model is run, the output window created by ArcMap can be used to track progress. Each tool validates the input and logs its execution status as text messages in the output window.

The Python tools expose their internal functions as an application programming interface (API) for a user to develop more sophisticated applications. The tools can also be run on the terminal (command console) by providing input as program arguments. This is useful when automating and scheduling routinely occurring workflows.

\subsection{Implementation Model}

Beneath the ArcMap GUI, the tools are chained together based on their input and output parameters to form a processing pipeline. The pipeline has four main stages. In the first stage, the input rasters are resampled to create a regular grid made up of points (nodes) and lines (edges). Resampling parameters like scale and geometry allow the user to control the coarseness of the grid, which affects the simulation time. In the second stage, the nodes and edges feature classes are loaded into an ArcGIS Network Dataset, which is a data structure used by ArcGIS Network Analyst to generate least-cost paths. The route simulations start in the third stage by creating multiple copies of the master network dataset and launching child processes to run the simulations with each process tasked to run a portion of the total number of simulations. Before running the least-cost path, each process first adds the uncertainty value to the network model and then runs Network Analysis's closest facility solver for generating least-cost paths between one of more source and destination points. At the end of each simulation, the process copies the generated route to its local database and continues with the next iteration. The simulation stops when each process has completed the prescribed number of routes. The original master process that created the child processes then gathers all the locally simulated routes and copies them to the routes feature class of the master database. After copying the resultant routes, the temporary databases are deleted and the simulated routes are plotted on the map. Figure 5-3 depicts the simplified program logic as a flowchart. 


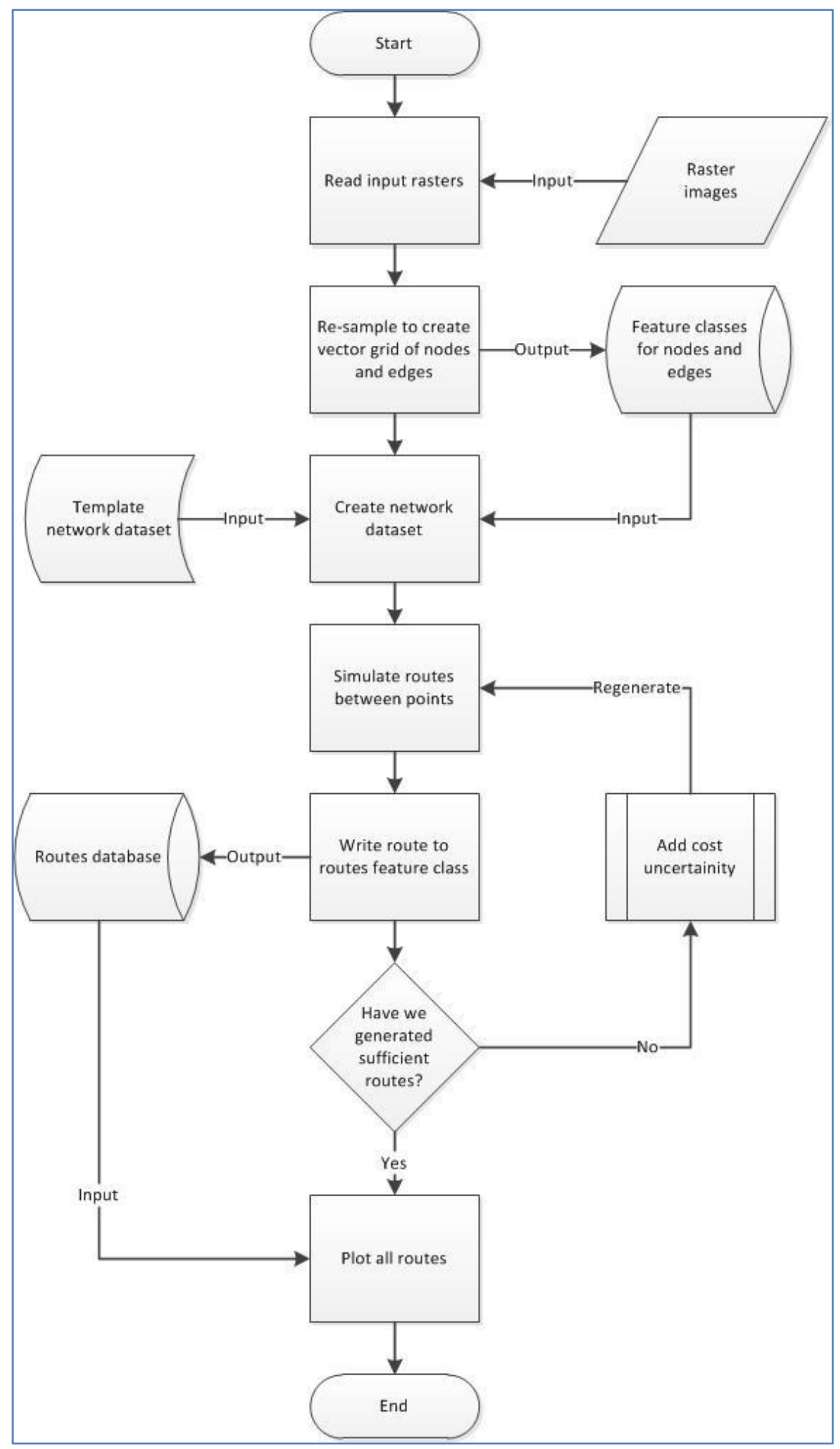

Figure 5-3: The program flowchart 


\subsection{Tools}

The program consists of four main Python scripts that use the arcpy library to perform geospatial operations. The tools are:

- Create Vector Layer

- Create Network Dataset

- Randomize

- Simulate Routes

\subsubsection{Create Vector Layer Tool}

This tool is responsible for resampling the input rasters and producing a vector grid of nodes and edges. The tool's main function accepts input from a terminal or through ArcMap's GUI. The set of rasters, geometry, and scale parameters are sent to the sample_raster_to_vector function which begins the resampling process by determining the coarsest raster from the input set. This is done by computing the resolution of each raster as a product of the cell width and cell height and then selecting the raster that has the coarsest resolution. The coarsest raster forms the basis of the vector grid. Two feature classes - one to hold the resampled points (nodes) and the other to hold the lines (edges) - are created and insert cursors are opened for both of them. The process of building the vector grid beings at the origin (first row and first column) and continues by creating a node at regular intervals. A utility class called RasterScanner was built within this tool to separate the task of reading the pixel value of the selected row and column so that a developer can easily extend this functionality. The RasterScanner object reads all the rasters in memory and returns the value at a particular row and column position by summing up the pixel values of all the rasters at that position. The resampled value returned by RasterScanner is assigned to the node's VALUE attribute in the nodes feature class. Figure 5-4 shows the structure of the sampled nodes. 


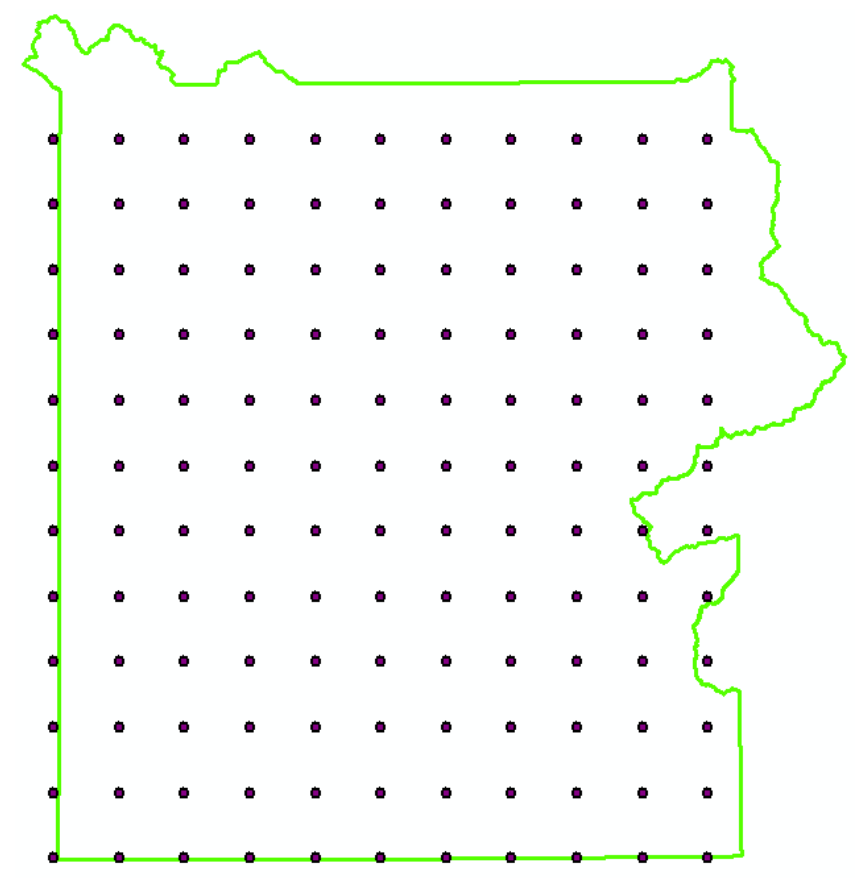

Figure 5-4: The resampling node structure

As the nodes are resampled, the edges connecting the nodes are created based on input geometry. For the Rook geometry, only horizontal and vertical edges between points are considered and added to the edges feature class. The cost attribute of the edge is defined as the difference in the values of the connecting nodes. For the Queen geometry (Figure 5-5), the diagonals between points are added to the edges feature class. The Knight geometry is the most complex by considering the diagonals between points that are two rows apart. 


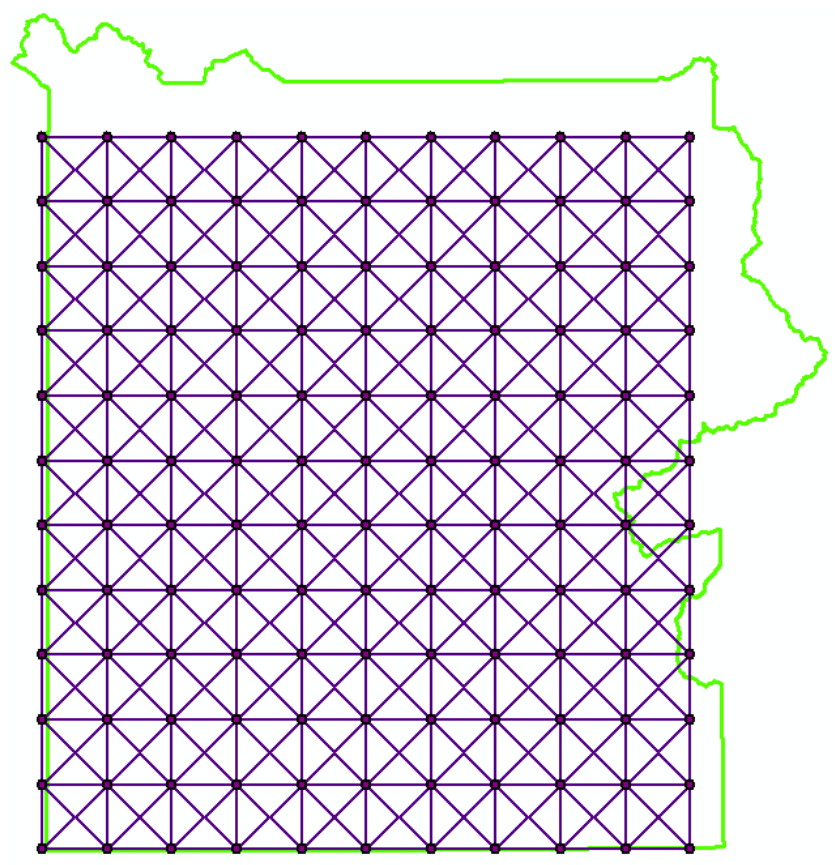

Figure 5-5: The vector model for Queen geometry

The resampling process stops once the last row and column (for the raster) are reached. The feature classes are closed and the insert cursors are released. This marks the end of the vectorization process.

\subsubsection{Create Network Dataset Tool}

At 10.1, it was not possible to create a network dataset in Python code. Therefore, a template network dataset was developed that contained the schema of the nodes and edges feature classes. The VegasTemplate file geodatabase contains the VectorModel feature dataset that has a network dataset within it. The network dataset was created through ArcMap with the cost attribute configured to use the COST field in the edges feature class. This is depicted in Figure 5-6. 


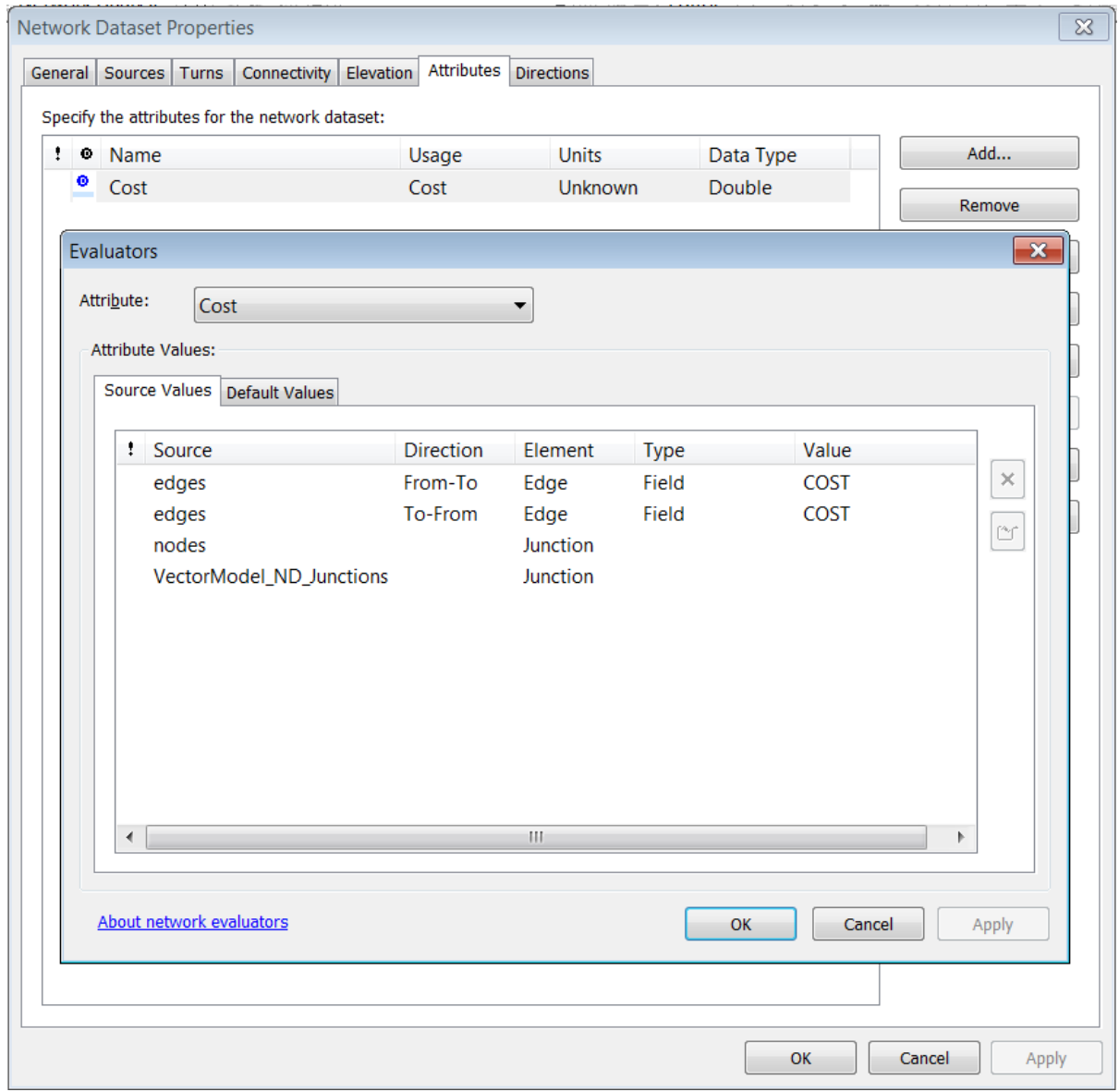

Figure 5-6: The creation of the template network dataset

The Create Network Dataset tool was then tasked to load the nodes and edges features created by the Create Vector Network tool into the template database. The tool achieves this by creating a new copy of the template database into the working directory. It then copies the nodes and edges feature classes using the Append tool in the geodatabase management toolbox in ArcMap. Once the copy is complete, the network dataset is rebuilt using the BuildNetwork Python function in the network analysis package of the 'arcpy' library. This requires that ArcMap be licensed with the Network Analyst Extension. If in the future versions of ArcMap the ability to create a network datasets is available in Python, this tool can be changed to use the new functions instead of a template dataset.

\subsubsection{Randomize Tool}

The randomize tool adds a user-defined noise value to the cost of the resampled nodes as a way to account for uncertainty and errors in reclassification of the input raster surfaces. The 10.1 version of network dataset did not allow for defining a network evaluator to add 
the noise value dynamically, therefore it was decided that the noise value would be added to the underlying nodes and edges feature classes before each simulation.

The randomize function in the tool takes as input the percentage of change to apply to the cost of each node, the path to the nodes and edges feature classes, and the path to the file geodatabase. The function first loads all the nodes in memory as a Python dictionary object and then applies the change by adding \pm change percentage to the value of the node. The function then iterates over every edge in the feature class and uses the backlink parameters of SRC_ID and DEST_ID attributes of each edge to identify the source node and destination node that make the edge. Once the two nodes are identified, the new cost of traversing the edge is computed as the difference in the node values and assigned to the edge's COST attribute. The new values of node and edges are once again written to their respective feature classes. Because the nodes and edges in the network dataset are updated with new values, the network dataset is rebuilt using the BuildNetwork function. Figure 5-7 represents the change in node values when a noise of $20 \%$ is applied to the nodes feature class through the Randomize tool.
00000000000
00000000000
00000000000
00000000000
00000000000
00000000000
00000000000
00000000000
00000000000
00000000000
00000000000
00000000000
- 0000000000
00000000000
00000000000
00000000000
00000000000
00000000000
00000000000
00000000000
00000000000
- 0000000000
00000000000
00000000000

Figure 5-7: Change in node values after running the Randomize tool

The Randomize tool is invoked by the 'Simulate Routes' tool between each simulation run of the least-cost path analysis.

\subsubsection{Simulate Routes Tool}

The Simulate Routes tool loads the source and destination points provided by the user into the master database created by the Create Network Dataset tool. To make the simulation efficient, the tool applies multi-processing by starting multiple worker processes based on the number of CPU cores specified by the user. In the 10.1 version of ArcMap, the file geodatabase could only support one writer process, although it could have many reader processes. To work around this limitation, the Simulate Routes tool created multiple copies of the master network dataset one for each of the worker 
processes (Figure 5-8). In doing so, the tools made sure that the database only had one writer process at a time.

\begin{tabular}{|c|c|c|}
\hline Name & Date modified & Type \\
\hline Degas.gdb & 10/7/2013 9:21 PM & File folder \\
\hline As worker0 & 10/7/2013 9:21 PM & File folder \\
\hline Derker1 & 10/7/2013 9:21 PM & File folder \\
\hline dis worker2 & 10/7/2013 9:21 PM & File folder \\
\hline du worker3 & 10/7/2013 9:21 PM & File folder \\
\hline Dorker4 & 10/7/2013 9:21 PM & File folder \\
\hline derker5 & 10/7/2013 9:21 PM & File folder \\
\hline D. worker6 & 10/7/2013 9:21 PM & File folder \\
\hline de worker7 & 10/7/2013 9:21 PM & File folder \\
\hline
\end{tabular}

\section{Figure 5-8: Multiple copies of the master database}

The multiprocessing package in Python provides APIs to create and interact with a pool of worker processes to perform tasks simultaneously. The Pool class takes in the number of worker processes to create and then allows the master process to define work for each worker process. The master process in the Simulate Routes tool defines work for the child processes through the simulate_for_worker function. This function begins by extracting the path for its work directory from the input provided by the master process. It then creates a new closest facility solver layer from the network analysis package and loads the source and destination points from the respective feature classes into it as facilities and incidents respectively. Each iteration of simulation in the worker processes invokes the Randomize tool to apply the noise value to the underlying network model, rebuilds the network dataset as the underlying costs have changed, and then solves the closest facility solver. Once the solver task has completed, the worker process keeps track of each edge within the solved route using the CopyTraversedSourceFeatures function in the network analysis package of the 'arcpy' library. A simple search cursor over the original edges feature class then increments the count of the traversed edge in the solved route. Figure 5-9 depicts the efficient use of an eight core CPU computer during simulation. 


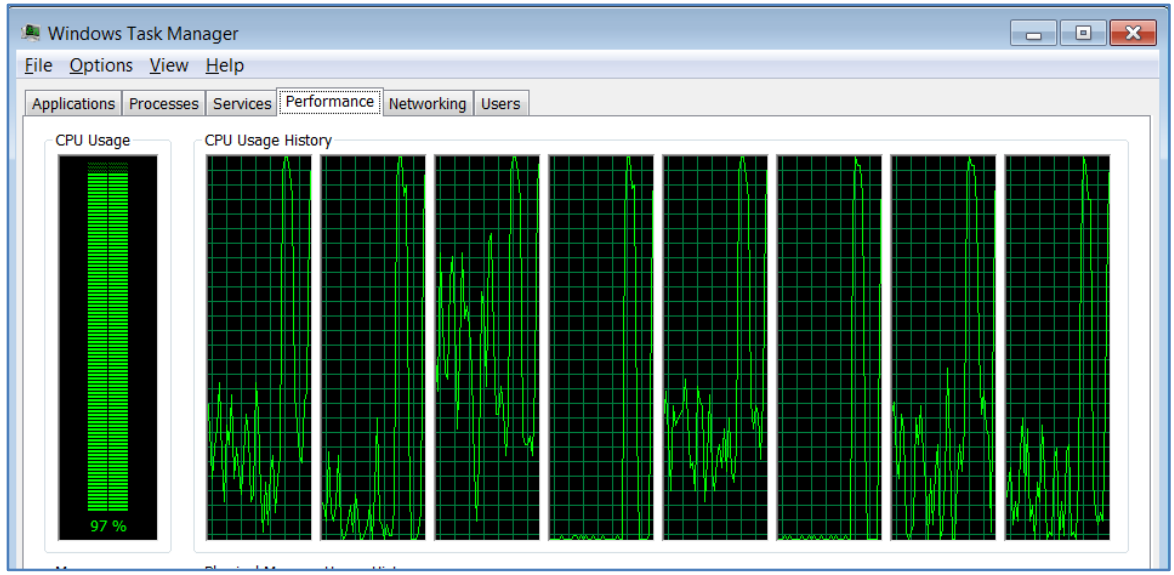

Figure 5-9: The CPU utilization during a simulation run with multi-processing

When all the worker processes complete their share of the simulations, the master process iterates over the routes feature class of every worker database and copies the path to the master database. Figure 5-10 shows the result of one complete simulation for 100 iterations using eight CPU cores. The source and destination points are depicted in green and maroon respectively. The edges in red have been traversed most frequently followed by edges in orange. The edges in blue are not traversed at all.

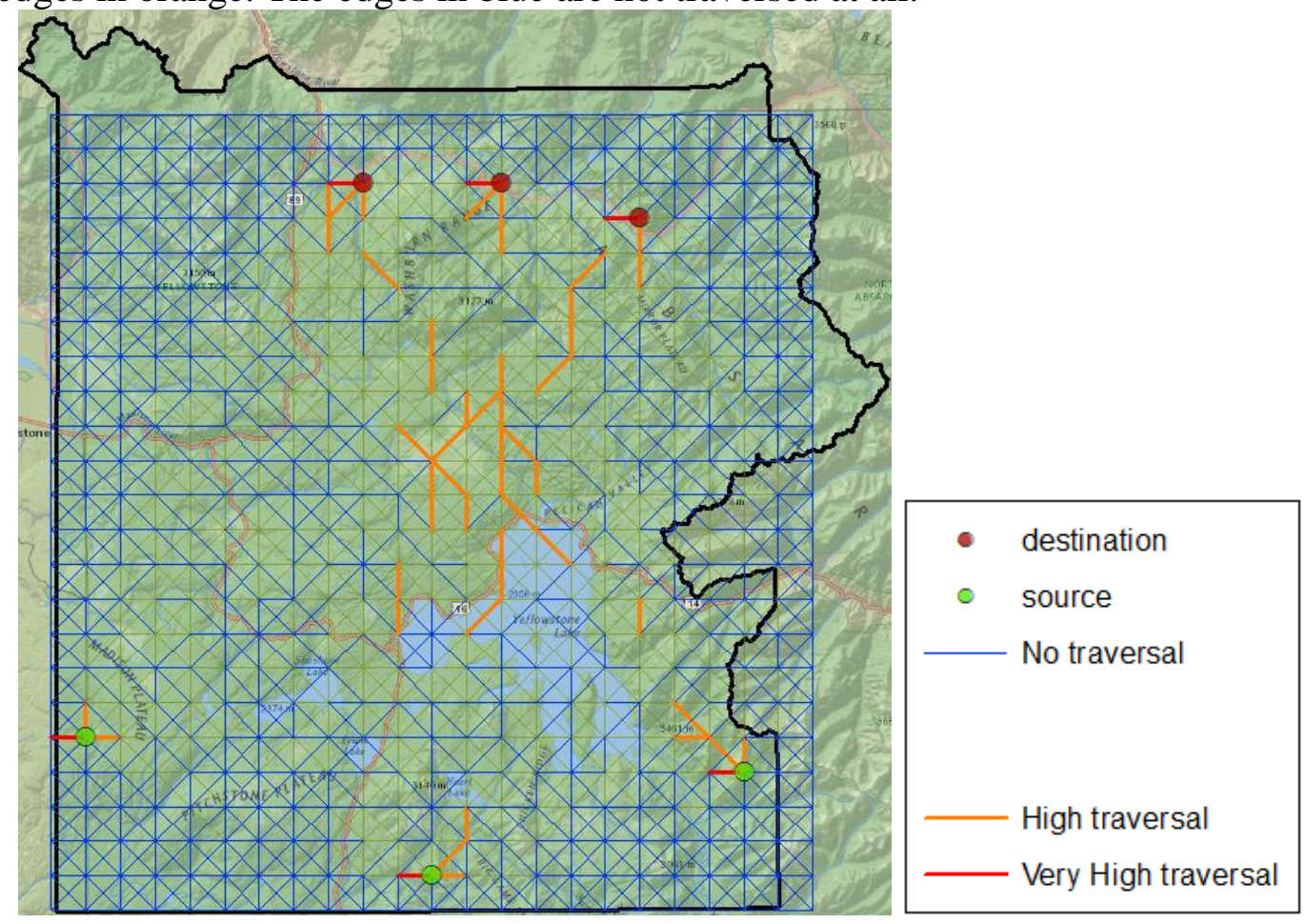

Figure 5-10: Result of simulation depicting most frequently traversed edges 


\subsection{Testing Methodology}

Testing and tool development went hand in hand to make sure that the functions developed in the tools met their functional requirements. The Yellowstone National Park dataset was used for unit testing. Every critical function in the tools was unit tested through Python's unit testing framework that set up the parameters required by the function, executed the function, and then validated the output of the function. For example, a raster image of $100 \times 100$ pixels, a scale of 10, and the Queen geometry was defined as the input to test the function that created the vector model. Once the vector model was constructed, the number of the nodes, and edges in the respective feature classes were validated based on the following formulae:

$$
\begin{aligned}
& \text { Nodes }_{\text {total }}=(\text { rows } / \text { scale }) \times(\text { cols } / \text { scale }) \\
& \text { Edges }_{\text {rook }}=2 \times\left(\text { Nodes }_{\text {rows }} \times \text { Nodes }_{\text {cols }}\right)-\text { Nodes }_{\text {rows }}-\text { Nodes }_{\text {cols }} \\
& \text { Edges }_{\text {queen }}=4 \times\left(\text { Nodes }_{\text {rows }} \times \text { Nodes }_{\text {cols }}\right)-3 \times \text { Nodes }_{\text {rows }}-3 \times \text { Nodes }_{\text {cols }}
\end{aligned}
$$

Once the tools were written and the unit testing complete, the tools were tested in a holistic fashion through the ArcMap GUI. The Yosemite National Park dataset was used for testing the platoon mobility scenario with different scales, input geometries, noise values, and number of CPU cores. During testing, it was found that the tools were constrained by the 32 bit Python runtime and could not process very large rasters.

\subsection{Automation and Scheduling}

It is important for a GIS analyst to be able to automate the tools so that they can be run on a regular schedule without any manual intervention. This is useful when the parameters applicable to the tools do not change often but the underlying data change frequently. The tools developed in this paper can be run on a terminal by providing input as command line arguments. When the tools are run on the terminal without any program arguments they are programmed to print out the tool help. This makes it possible to automate the tools and workflow with the help of a scheduler application like 'at' in UNIX or 'Task Scheduler' in Windows. The functions in the Python scripts can also be used as APIs to build other scripts that need some of the tool's core functions.

\subsection{Summary}

The above sections describe the tool implementation. The tools were bundled in an ArcMap toolbox and delivered to the client along with the source code. Testing the tools during development helped in debugging out a large number of bugs early on. The next chapter describes the tools use case and the impact of each input parameter to the simulation. 


\section{Chapter 6 - Results and Analysis}

Chapter 6 studies the tool applications and presents a comparative analysis of tool's input. Section 6.1 presents the lost platoon mobility scenario in which a GIS analyst is tasked with developing a plan for the traversal of an infantry platoon across a terrain. Starting from Section 6.2, the impact of each of the input parameters is described in detail. Section 6.6 presents the chapter summary.

\subsection{Platoon Mobility Scenario}

The mobility modeling tools presented in this paper were specifically developed as an aid in the planning of traversal routes for an infantry platoon. The goal of the planning operation was to find optimal routes for the platoon to cross the terrain by evading the enemy and preventing any ambush. It was therefore important to find out areas of the terrain that could become possible ambush points. For the hypothetical use case, it was assumed that during the planning phase all information regarding the terrain was available beforehand (with some uncertainty) and that the platoon had a clear starting and ending locations.

The first step was to gather geospatial data on the factors that influence traversal on the terrain such as slope, aspect, land use, presents of roads and highways, well-known trails, and points of interest on the terrain. The data were scrubbed and loaded into a file geodatabase and the vector datasets were converted to raster images. Every raster image was then reclassified with lower values assigned to areas in the raster that were favorable to traversal. For example, in the slope raster during reclassification, lower values were assigned to areas of gentle slope using the rationale that the platoon would prefer to traverse over a gentler terrain. Once all the raster images were reclassified using local knowledge or prior experience, they were combined to create a final cost surface for the terrain. The starting points of the platoon were treated as source points and the final destination were treated as destination points. To begin the simulation, a larger scale and noise percentage value were to identify the approximate areas of bottlenecks. The values were refined for each of the subsequent runs to focus on more specific regions on the terrain.

Each run of the simulation produced a feature class that contained all the simulated routes between source and destination points. The simulated routes were plotted on a map to understand the general trend of traversal. To identify bottlenecks (possible ambush points), the 'REPEATED' attribute in the edges feature class was symbolized using a graduated color palette as shown in Figure 6-1. 


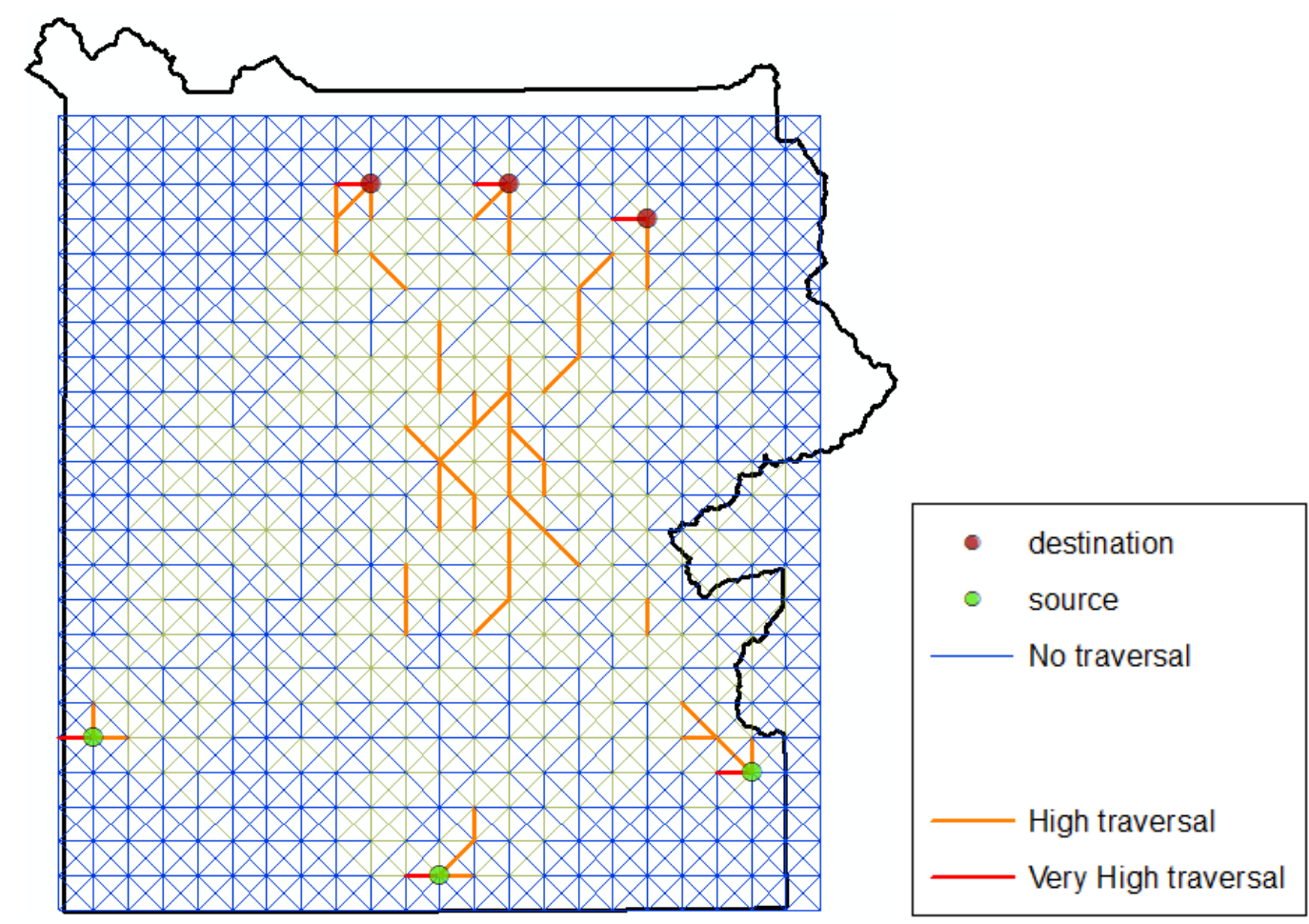

Figure 6-1: Most frequently traversed edges between source and destination points

\subsection{Impact of Scale Variation}

The scale parameter in the program input affects the resampling resolution when the raster cost surface is converted to a vector grid. A smaller scale value closely reflects the underlying cost surface, producing accurate routes. However, it increases the number of nodes and edges in the network model, leading to an increase in the computation time. Table 5 presents the effect of scale variations on the accuracy and the time taken to complete a fixed number of simulations. The data were generated on the Yellowstone National Park dataset for 100 iterations on a four core CPU with the randomization value set to be at $10 \%$. The accuracy was measured in terms of the length of horizontal and diagonal edges.

Table 5. Effect of scale variations on accuracy and computation time

\begin{tabular}{|r|r|r|r|r|r|}
\hline Scale & No. of nodes & No. of edges & $\begin{array}{l}\text { Length of } \\
\text { horizontal } \\
\text { edge (miles) }\end{array}$ & $\begin{array}{l}\text { Length of } \\
\text { diagonal edge } \\
\text { (miles) }\end{array}$ & $\begin{array}{l}\text { Time } \\
\text { (minutes) }\end{array}$ \\
\hline 5 & 2303 & 8926 & 1.3 & 1.83 & 44 \\
\hline 10 & 552 & 2069 & 2.6 & 3.69 & 9.40 \\
\hline 15 & 240 & 869 & 3.9 & 5.53 & 4.34 \\
\hline 20 & 132 & 461 & 5.35 & 7.38 & 3.11 \\
\hline
\end{tabular}


In Figure 6-2, it is evident that the increase in computation time is almost exponential with respect to the value of the scale used for simulation. To speed up computation time, a faster computer with a larger number of CPU cores may be required. Another option is to use a larger scale value for larger extents. The simulations can be rerun with smaller scale values on focused areas of the terrain that are more susceptible to bottlenecks.

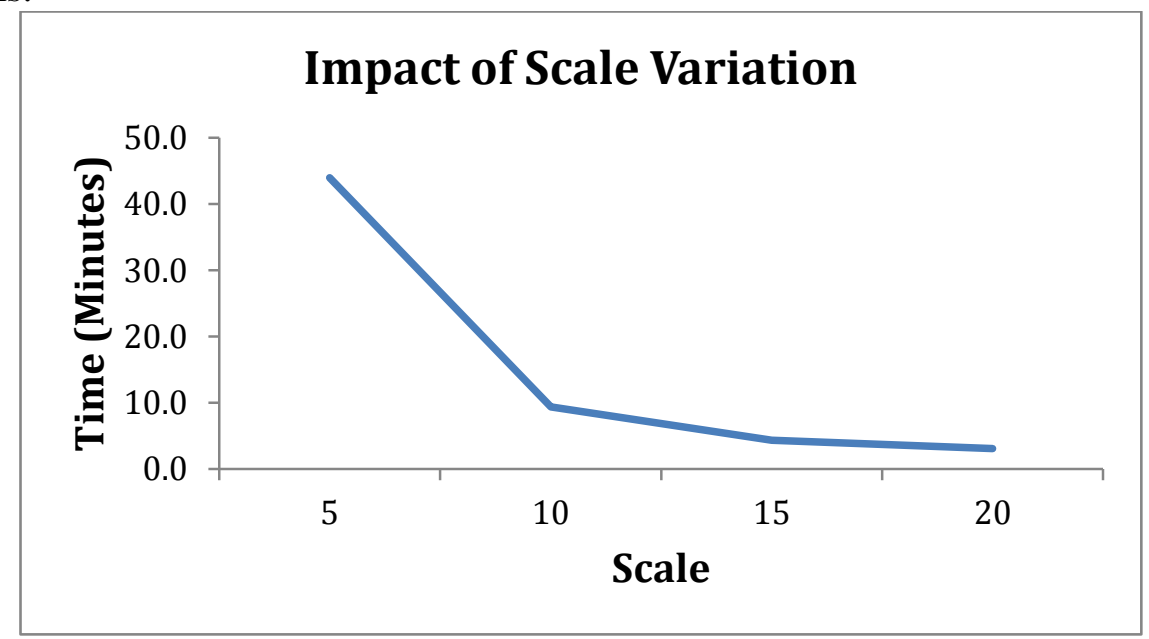

Figure 6-2: A graph of scale vs. computation time (in minutes)

\subsection{Impact of Noise Variation}

The randomization parameter is designed to add noise to a route simulation to account for errors and uncertainty in measurement of the cost surface. By adding noise to the underlying cost surface, sub-optimal routes between source and destination are considered for analysis. While a noise value between five and 20 percent would be considered typical, a GIS analyst can apply local knowledge about the terrain and decide on a suitable value. Figure 6-3 depicts the difference in the popular routes when a noise value of five and 20 percent are compared with the optimal route between two points. It is evident that when a large noise value is configured for simulation, routes farther away from optimal are considered. 


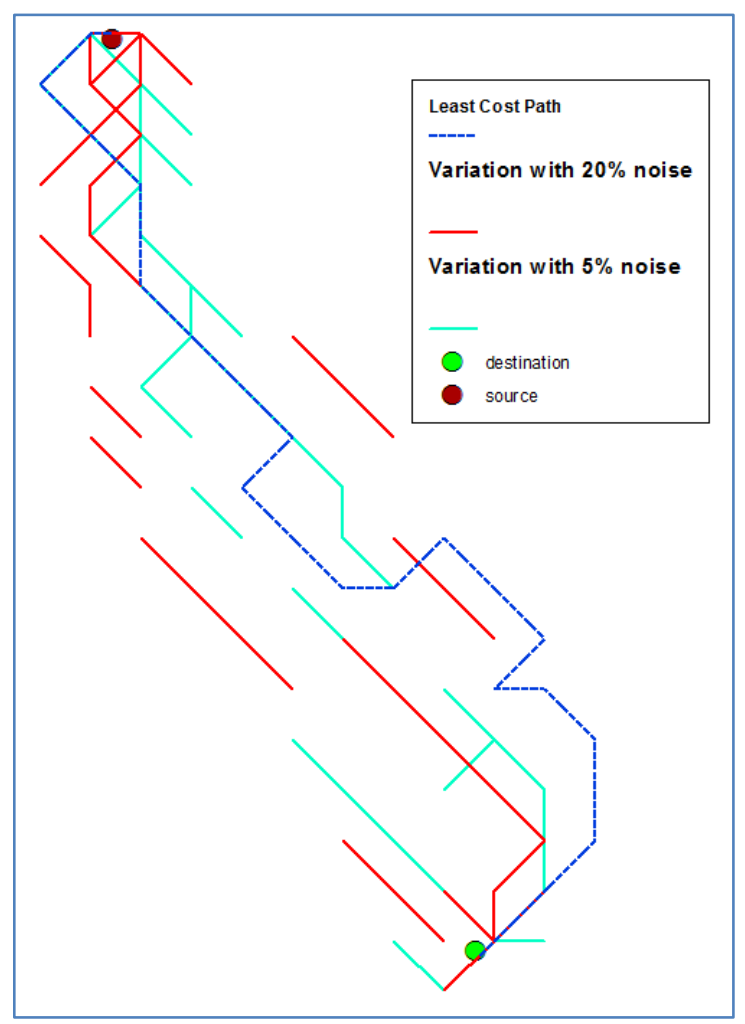

Figure 6-3: Impact of noise on simulation

\subsection{Impact of CPU Cores}

By changing the number of CPU cores parameter, one can control the degree of parallelization for the route simulation. The greater the degree of parallelism, the shorter will be the computation time for a given set of iterations. Optimum performance is achieved by setting the number to the number of CPU cores available on the computer. This configuration guarantees that every process running the simulation has a CPU core available to do its work, reducing any latency introduced by waiting for the availability of a CPU. However, setting the value of this parameter to greater than the number of available cores can adversely affect performance as there will be contention for the CPU. Figure 6-4 plots the graph of the number of CPU cores parameter with the time taken to complete the simulation. 


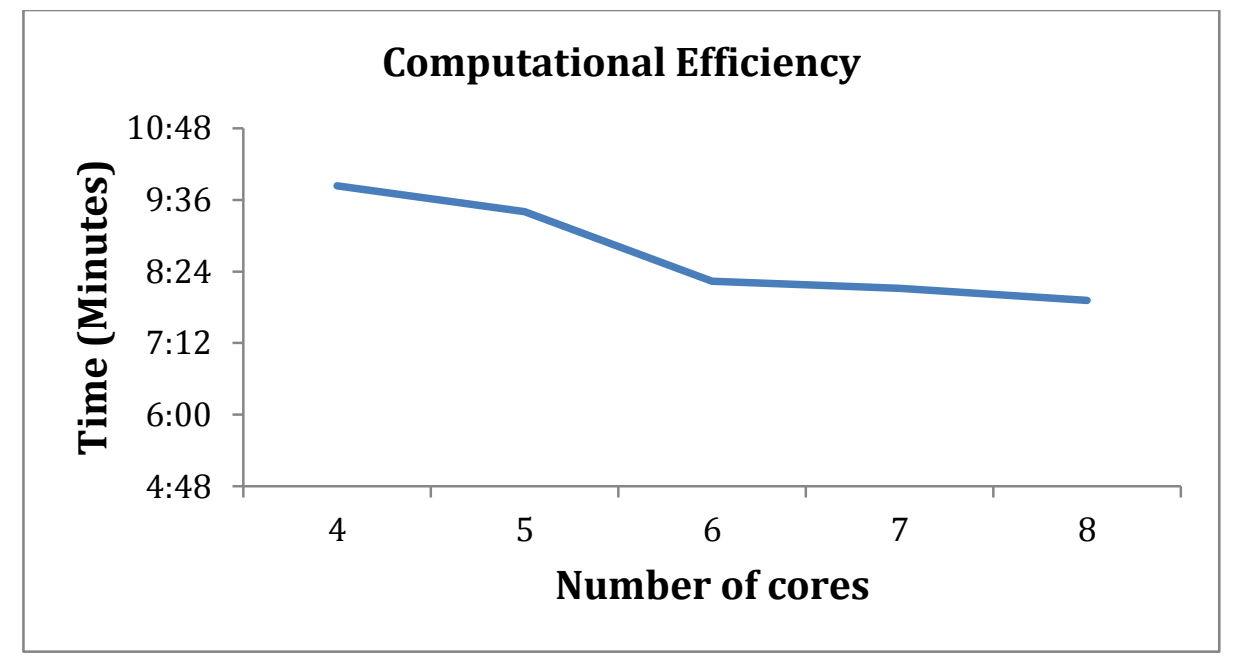

Figure 6-4: Impact of the number of cores on computation time (minutes)

\subsection{Impact of Terrain Model}

The most important element in the analysis of human mobility is the underlying cost surface. A cost surface model can include one or more geospatial factors such as elevation, aspect or land-use and is typically developed to solve a specific problem. Local knowledge or prior experience in the region can be applied to the cost surface by adding specific biases. For example, a directional bias can be applied by creating a raster surface that has low costs in a small aspect range. Depending on the nature of the problem, whether it is enemy detection or avoidance, time of day and geospatial features on a terrain like tall peaks or deep ravines become important. Non-spatial factors such as average age of the group moving across the terrain, height of individuals, or the equipment carried by them can be taken into consideration while developing the surface model. For example, a large water body can be assigned high costs when individuals are traversing on foot. However, the same water body can be traversed fairly easily with boats.

The individual factors can be combined in a weighted fashion when certain factors are more important than others. The simulation tools developed in this paper perform a simple overlay sum when presented with more than one raster surface to produce the resampled vector grid.

\subsection{Strength of the Analysis}

For the analysis to be useful it must be consistent across runs. To measure the consistency, the simulation was run 3 times with 100 iterations in each run. From each run, the edges that were traversed more than 20 times were highlighted and plotted on a map. The comparative results are depicted in figure 6-5. The map clearly indicates that even though each run performs Monte Carlo simulation, the most frequently traversed 
edges from each run are very close to each other highlighting the consistency in the analysis.

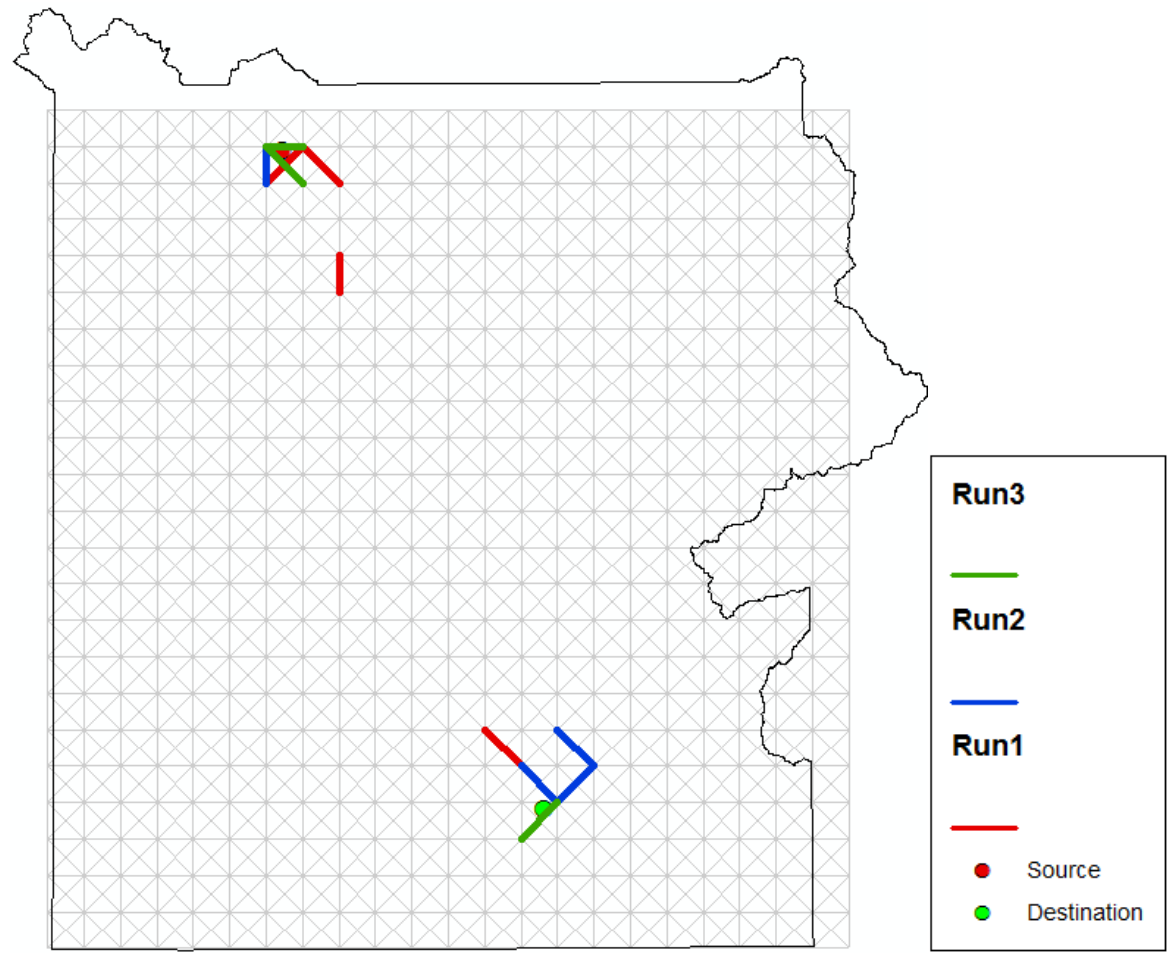

Figure 6-5: Comparison of 3 simulation runs of the tool

\subsection{Normalizing Diagonal Costs}

Based on the client's requirement, the cost of traversing an edge was computed as the difference in values of connecting nodes. However, this cost did not account for the length of edge, causing the cost a vertical or horizontal edge to be same as the cost of a diagonal edge. This resulted in the least-cost path algorithm preferring diagonal edges over horizontal or vertical edges. To correctly account for the length of the edge, the costs must be normalized based on the edge length. Figure 6-6 presents the result of route simulation on un-normalized edge costs over normalized edge costs. The figure clearly indicates that when diagonal length was not accounted in the costs, the route simulation preferred diagonal edges resulting in more traversal edges along the diagonals. 

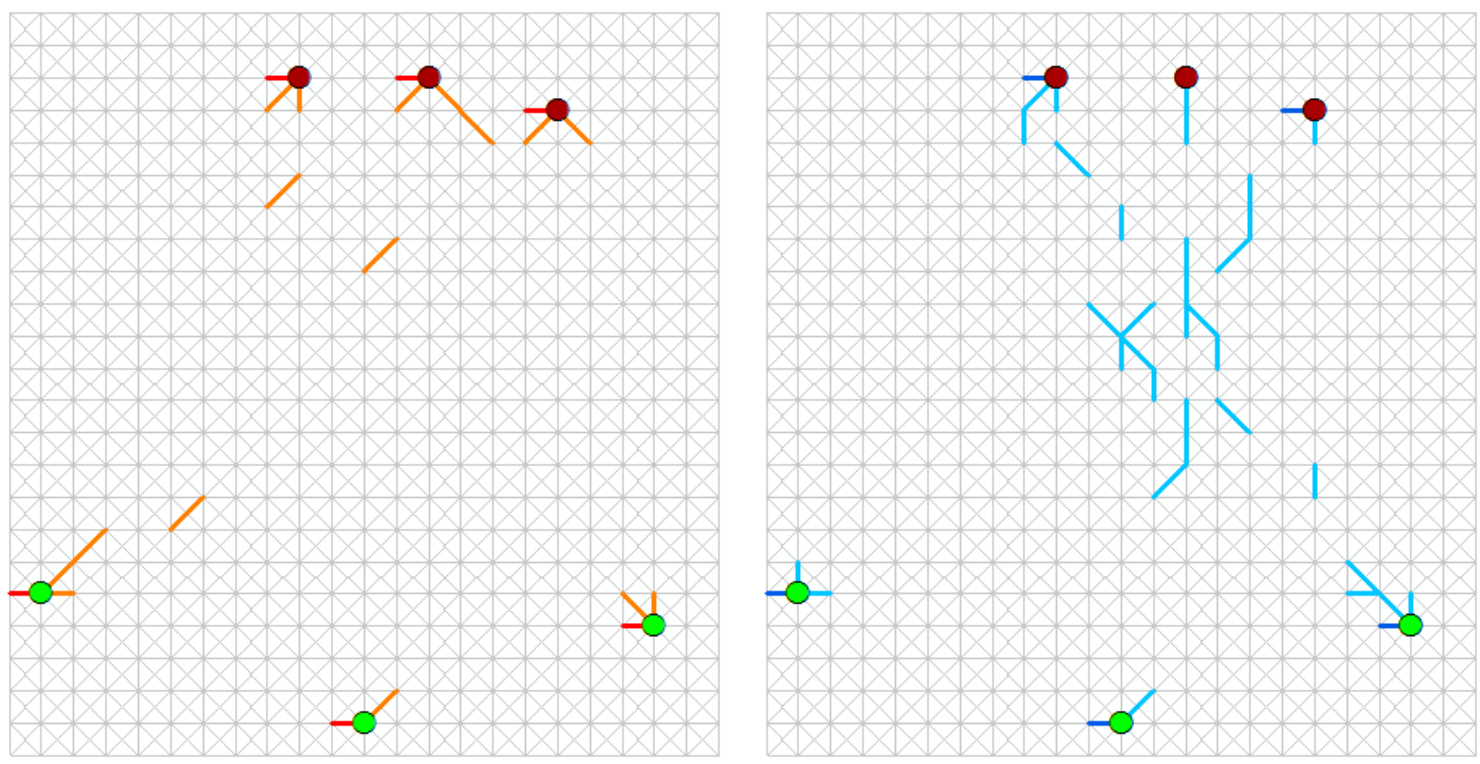

Figure 6-6: Comparing routes with un-normalized costs (left) with normalized costs

\subsection{Summary}

This chapter describes the various elements of the simulation tools that can be adjusted to meet the needs of a particular analysis. Of all the factors, the creation of the cost surface model forms the basis of decision making and path selection, making it a vital component in route simulation. The resolution and accuracy of the analysis can be controlled by the scale and noise parameters, whereas the number of CPU cores parameters affects the time taken for the simulation process. In addition to the out-of-the-box functionality, the simulation tools also provide extensibility points for further development and application. This is the topic of discussion in Chapter 7. 


\section{Chapter 7 - Conclusions and Future Work}

This chapter presents the conclusion and future work for the vector based mobility modeling tools. Section 7.1 summarizes the work and the project's final deliverables to the client. While the project met the goals set for it, the implemented can be improved and optimized. The future work and possibilities are described in Section 7.2.

\subsection{Conclusion}

The goal of the project was to develop a set of portable tools that could be used to model human mobility over a terrain. The tools were developed as an aid for analysts and planners who were tasked with developing rescue missions or to manage the logistics of troop movement for security and defense purposes. While the tools could not be tested using client data, they met the client's functional and operational requirements in solving the hypothetical problem of planning a route for an infantry platoon moving across a terrain. The tools were able to successfully highlight bottlenecks on a terrain by simulating a large number of routes on a cost surface within a short amount of time.

The tools apply the techniques of multi-criteria evaluation, least-cost path analysis and simulation to highlight optimal and sub-optimal routes between two points. However, they rely heavily on the GIS analyst to develop an intelligent and accurate cost surface model for the study region. Every cost model must be developed to solve a specific problem and must take into account physical, environmental and geospatial factors.

During the delivery phase of the project, the simulation tools were demonstrated to the client for final approval. The final deliverables to the client consisted of the Python source code for the tools, the geoprocessing toolbox for ArcMap, development and testing datasets, and a training manual that explains the installation, usage, and the inner workings of the tool.

\subsection{Future Work}

The mobility modeling tools create a vector model from the raster-based cost surface using a simple resampling technique. The resampling function takes into account only a single pixel for resampling, making the model susceptible to noise. A robust version of the resampling can be developed that applies a more sophisticated algorithm like kernel density or neighborhood averaging to produce the vector model. This can be achieved by changing the Python code in the Create Vector Model script.

To leverage the ArcGIS Network Analyst extension for route simulation, a network dataset had to be constructed from the vector model. However, in the 10.1 version of the software, it was not possible to create a new network dataset from scratch through Python scripts. Therefore, a pre-created template network dataset was shipped with the project to aid in network dataset creation. In the future, it may be possible to build a network dataset from Python code with complex cost evaluators, making the template dataset obsolete.

Though the tools use multi-processing to speed up simulation, they are bounded by the number of CPU cores on a single machine. By porting the modeling tools to ArcGIS 
Server, the tools can benefit from the server's 64 bit Python runtime and its ability to distribute the work to available server nodes, making the simulation even more efficient. ArcGIS Server also has the capability to web enable the geoprocessing tools, making them accessible to a larger audience through web services.

The least-cost path analysis to find optimal paths did not take into account the time of day or other restrictions for traversal. However, the network analysis extension has a large number of provisions to model practical constraints like time of day, capacity of edges and turn restrictions. These provisions can be exploited by the tools to produce a more accurate analysis of the terrain.

The tools were also developed with the intention that they can be used as APIs within larger programs. Every script is modular and organized into coarse-grained functions to perform a focused task. The Python scripts can be chained together to build a sophisticated predictive model for human traversal by embedding statistical and probabilistic tools and analysis. 


\section{Works Cited}

Andrew, W. (2012). A Prototype Path Prediction Tool. Redlands, California: University of Redlands.

Bagli, S., Geneletti, D., \& Orsi, F. (2011). Routeing of power lines through least-cost path analysis and multicriteria evaluation. Environmental Impact Assessment Review, 234-239.

Bhattacharya, P., \& Gavrilova, M. L. (2007). Voronoi diagram in optimal path planning. 4th International Symposium on Voronoi Diagrams in Science and Engineering (pp. 38-47). Glamorgan: IEEE.

Collischonn, W., \& Pilar, J. V. (2000). A direction dependent least cost path algorithm for roads and canals. International Journal of Geographical Information Science, 397-406.

DelSanto, K. (2011). Using Graph Theory to Outsmart Opponents.

Dikjstra, E. W. (1959). A Note on Two Problems in Connexion with Graphs. Numerische Mathematik, 269-271.

Garrido, S., Moreno, L., Abderrahim, M., \& Martin, F. (2006). Path Planning for Mobile Robot Navigation using Voronoi Diagram and Fast Marching. International Conference on Intelligent Robots and Systems (pp. 2376 - 2381). Beijing: IEEE.

Jahanpeyma, M., Delavar, M. R., Malek, M. R., \& Kamalian, N. (2011). Analytical Evaluation of Uncertainty Propagation in Seismic Vulnerability Assessment of Tehran Using GIS. Journal Civil Eng Urban, 05-09.

Kumler, M. P. (1999). Thiessen's Remarkable Polygons. California Geographical Society. 
Murrieta-Flores, P. A. (2010). Traveling in a Prehistoric Landscape: Exploring the Influences that Shaped. 37th International Conference on Computer Applications and Quantitative Methods in Archaeology (pp. 249-267). Williamsburg: Archaeopress.

Okabe, A., Boots, B., Sugihara, K., \& Chiu, S. N. (1992). Spatial Tessellations: Concepts and Applications of Voronoi Diagrams. John Wiley \& Sons, Ltd.

Sethian, J. A. (1998). Fast Marching Methods and Level Set Methods for Propogating Interfaces. von Karmon Institute Lecture Series, Computational Fluid Mechanics.

Thiessen, A. H., \& Alter, J. C. (1911). District No. 10, Great Basin. Monthly Weather Review, 1082-1084.

Zhou, G., Esaki, T., Mitani, Y., Xie, M., \& Mori, J. (2003). Spatial probabilistic modeling of slope failure using an integrated GIS Monte Carlo simulation approach. Engineering Geology, 373 - 386. 


\section{Appendix A. Analysis of the Yellowstone Dataset}
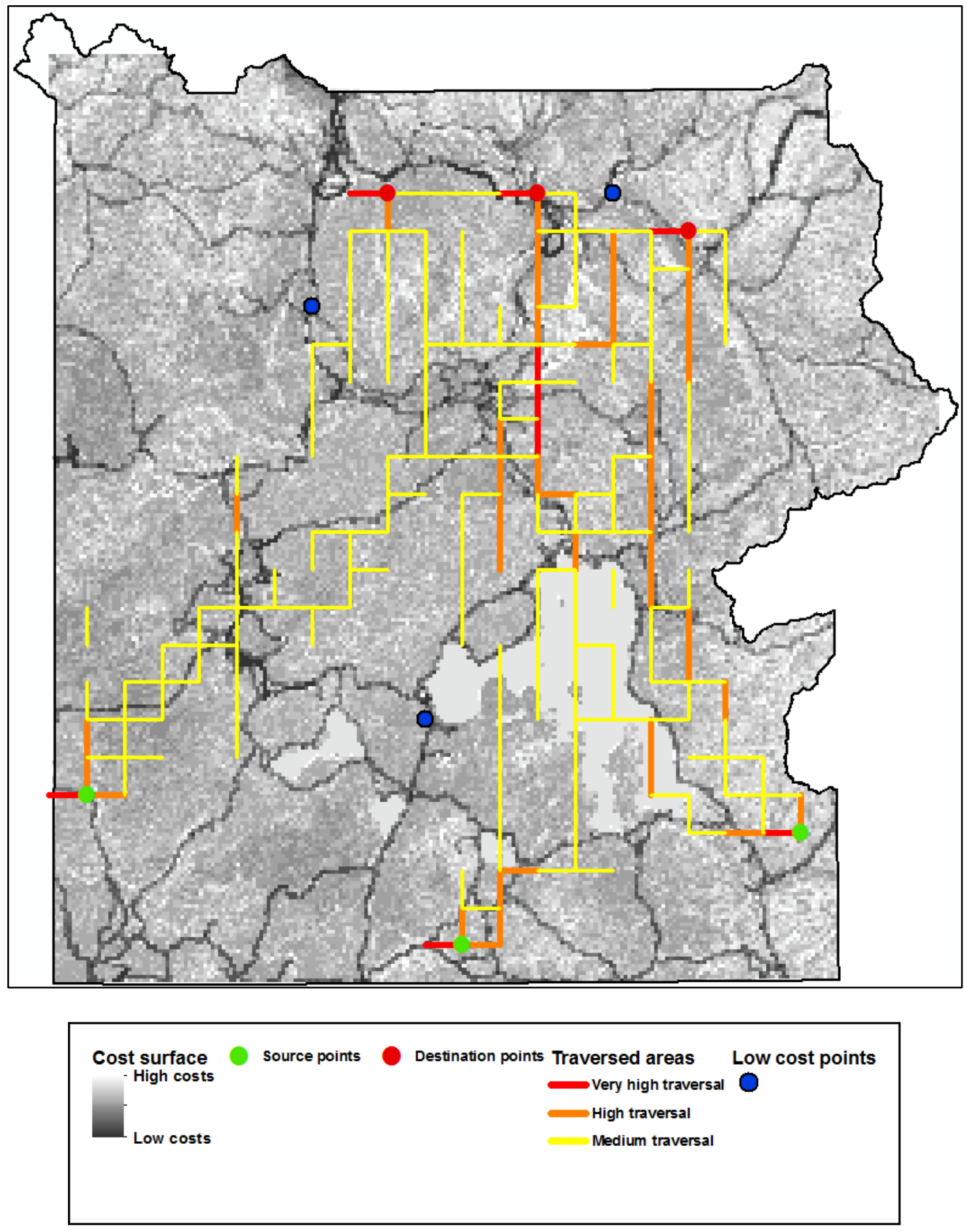

Figure 1: Rook geometry with scale value of 10 

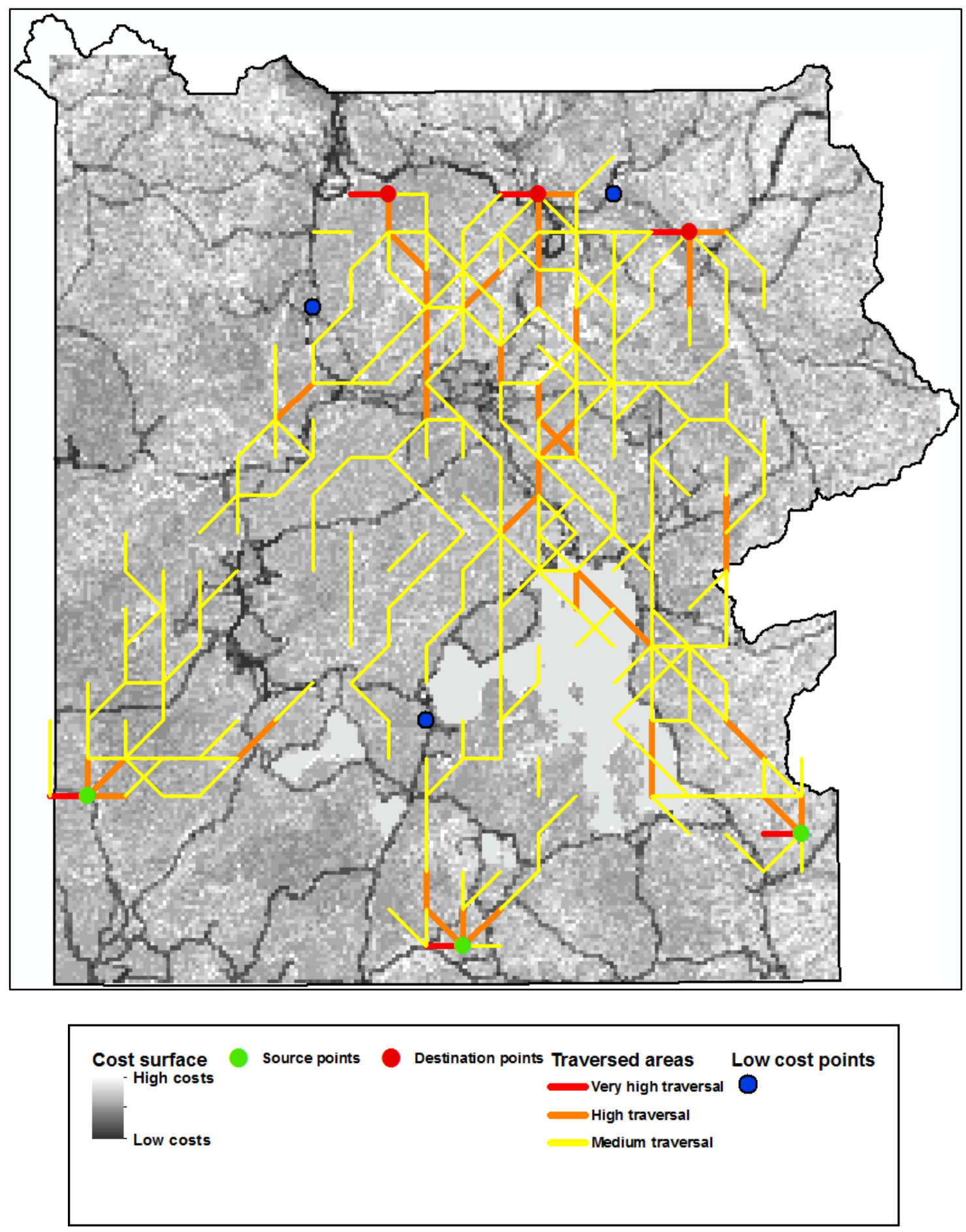

Figure 2: Queen geometry with scale value of 10 

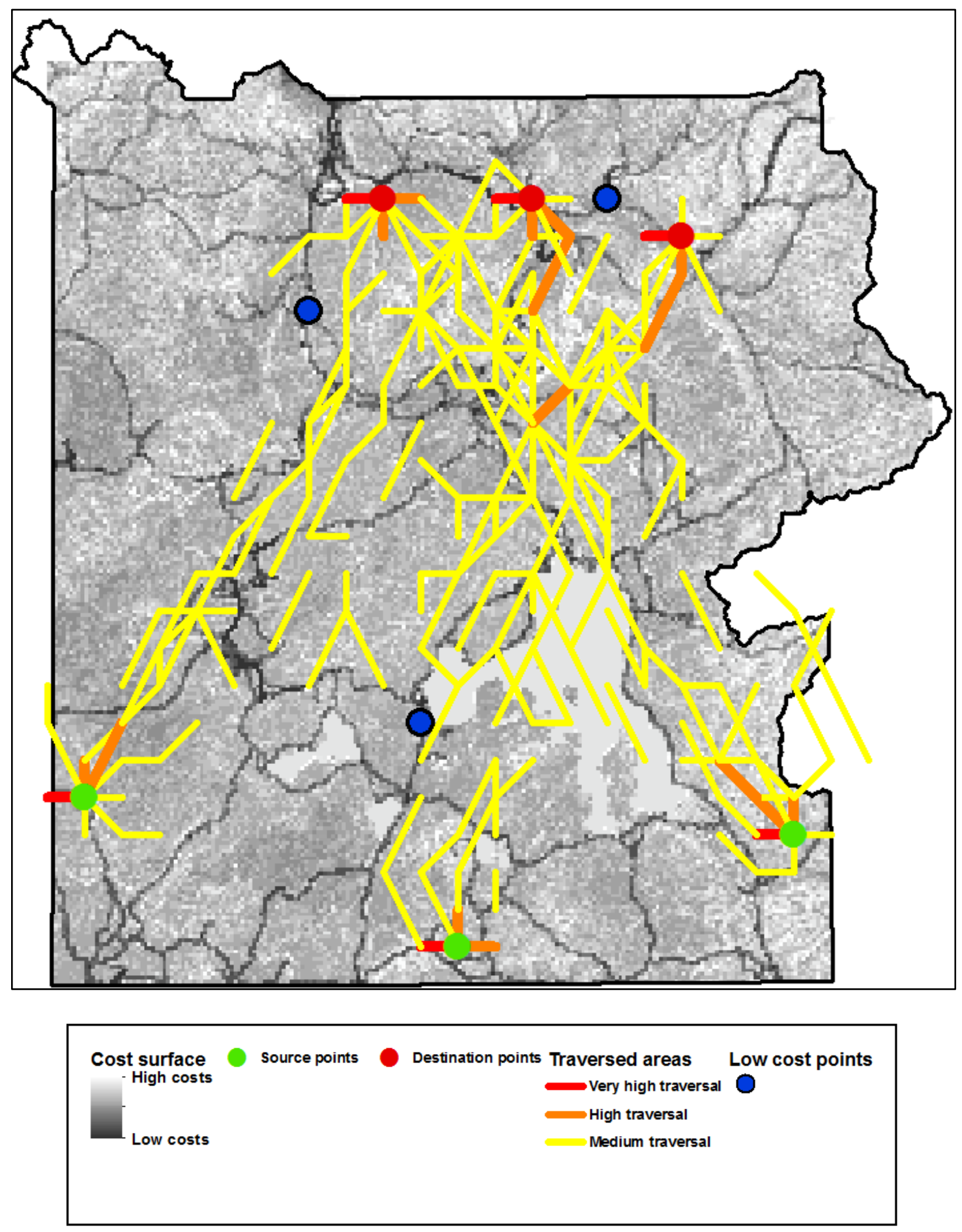

Figure 3: Knight geometry with scale value of $\mathbf{1 0}$ 


\section{Appendix B. Analysis of the Yosemite Dataset}
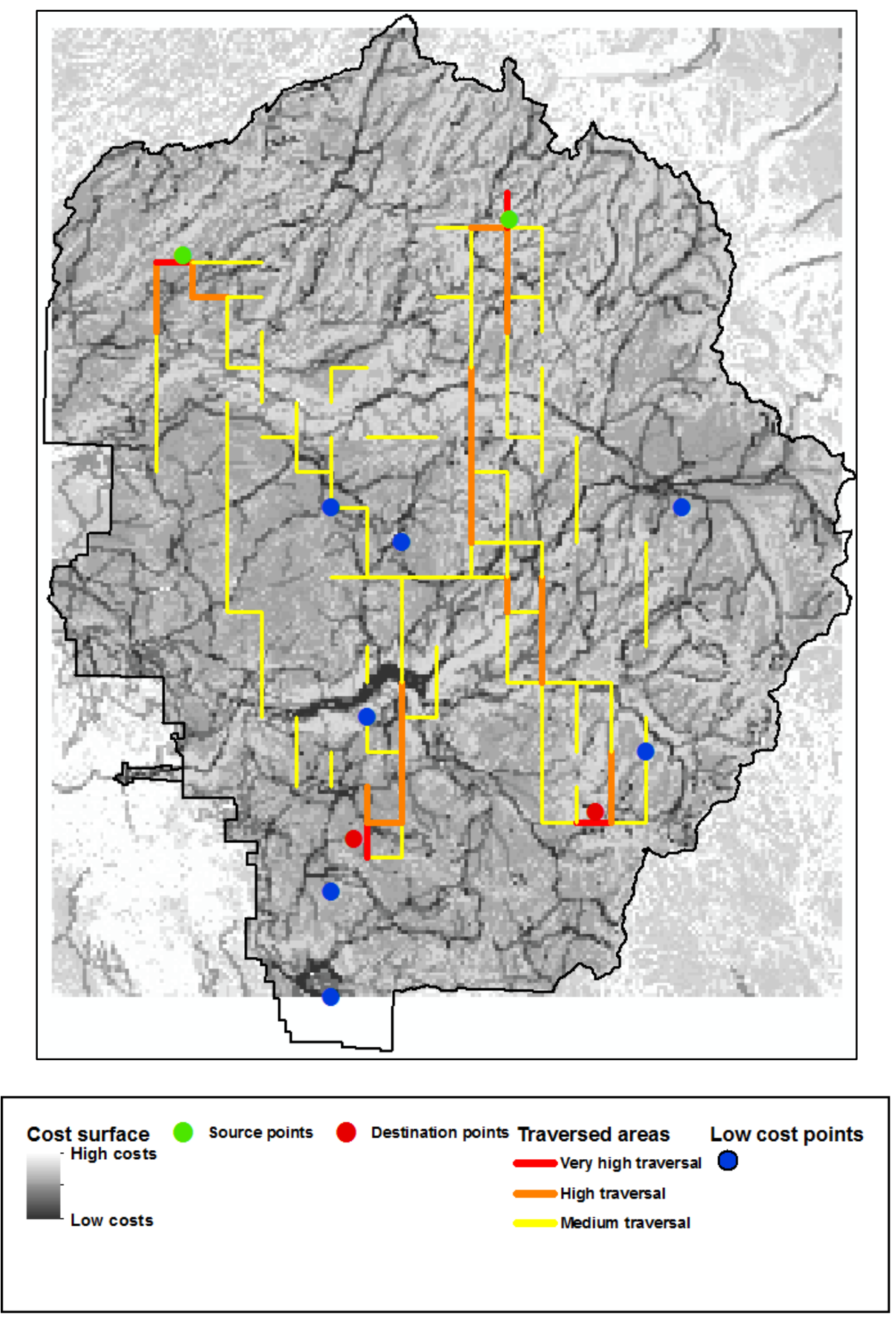

Figure 4: Rook geometry with scale value of 10 

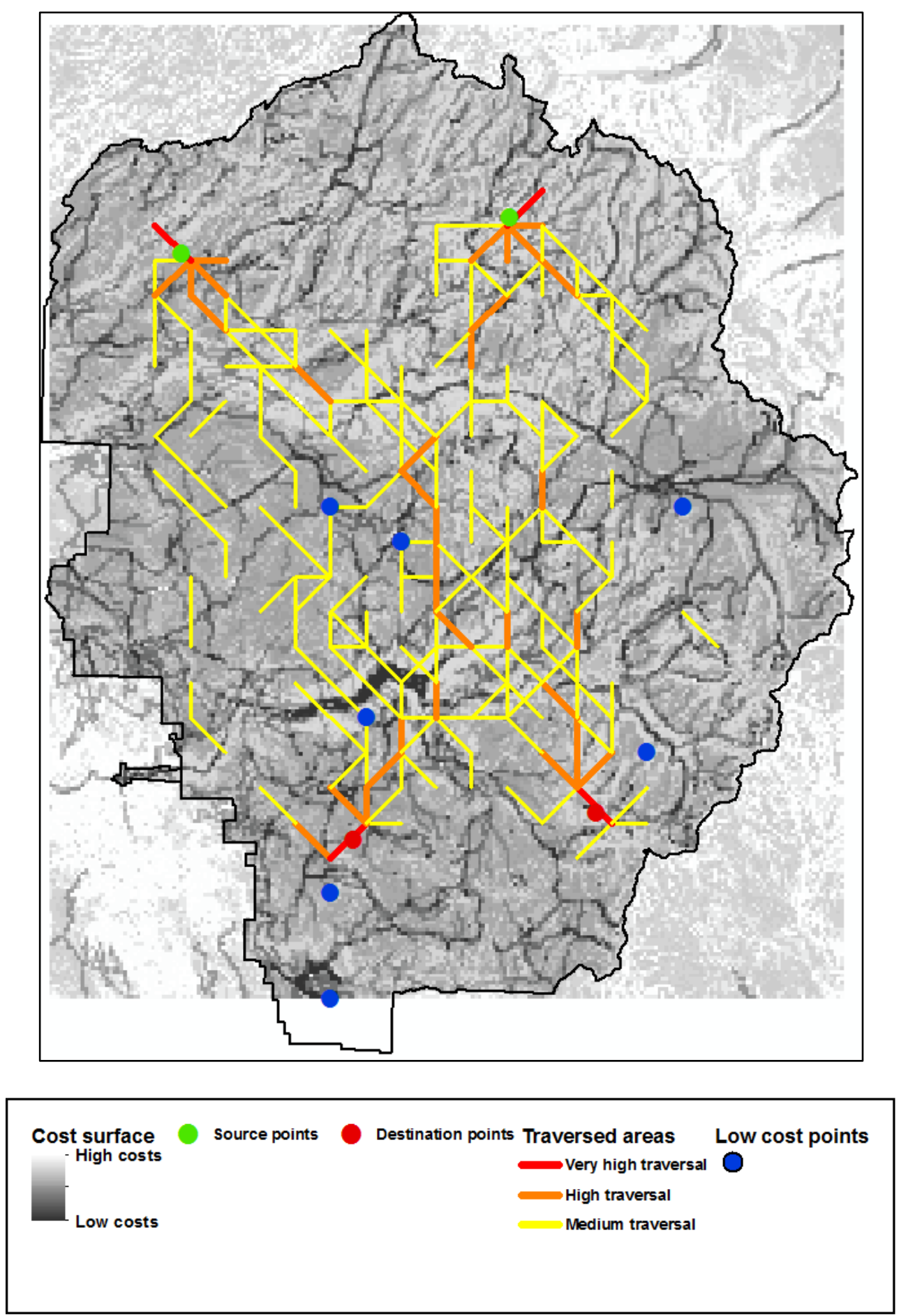

Figure 5: Queen geometry with scale value of 10 

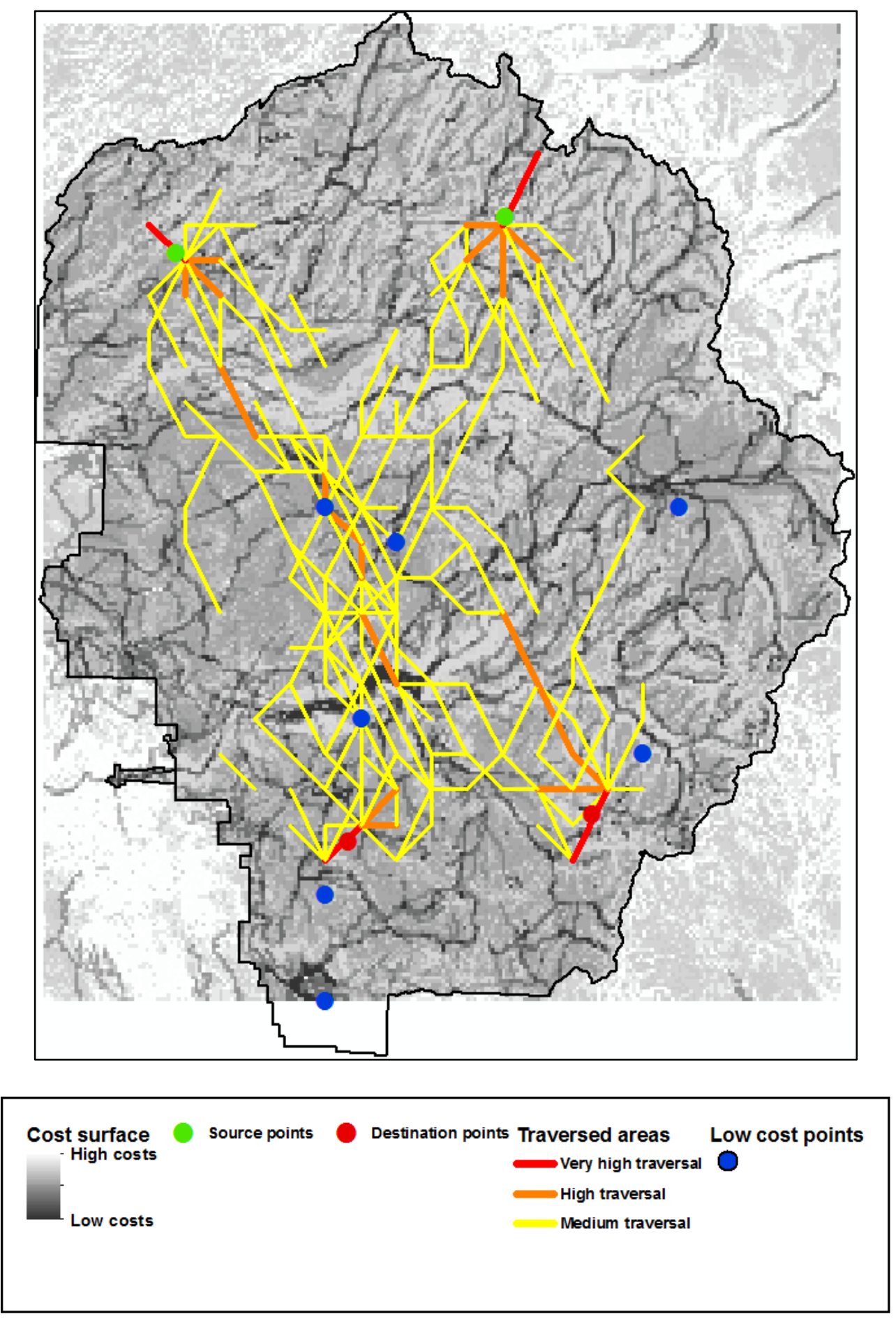

Figure 6: Knight geometry with scale value of $\mathbf{1 0}$ 